NISTIR 6901

\title{
Thermal Performance of Fire Fighters Protective Clothing. 2. Protective Clothing Performance Simulator - User's Manual
}

Kuldeep Prasad Hoang Minh Ho Dac

Scott Kukuck 


\title{
Thermal Performance of Fire Fighters Protective Clothing. 2. Protective Clothing Performance Simulator - User's Manual
}

\author{
Kuldeep Prasad \\ Hoang Minh Ho Dac \\ Scott Kukuck \\ Fire Research Division \\ Building and Fire Research Laboratory \\ National Institute of Standards and Technology \\ Gaithersburg, MD 20899-8660
}

January 2003

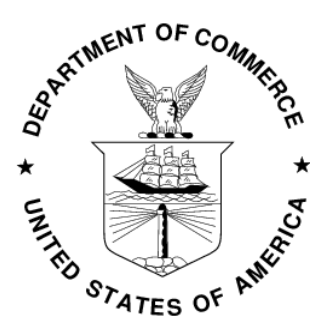

\section{U.S. DEPARTMENT OF COMMERCE}

Donald L. Evans, Secretary TECHNOLOGY ADMINISTRATION

Phillip J. Bond, Under Secretary of Commerce for Technology NATIONAL INSTITUTE OF STANDARDS AND TECHNOLOGY Arden L. Bement, Jr., Director
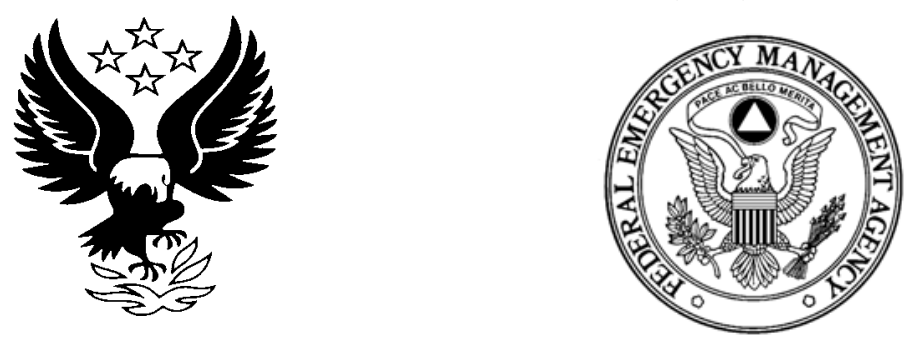

Sponsored in part by:

FEDERAL EMERGENCY MANAGEMENT ADMINISTRATION

Joe M. Allbaugh, Director U.S. FIRE ADMINISTRATION R. David Paulison, Administrator 


\section{Table of Contents:}

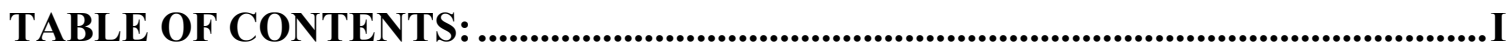

CHAPTER 1: INTRODUCTION AND INSTALLATION OF PCPS......................... 1

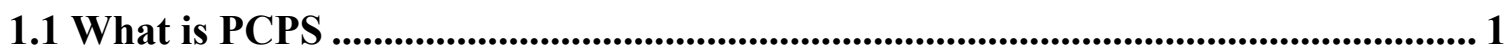

1.2 System Requirements ............................................................................................. 2

1.3 How to install PCPS......................................................................................................... 2

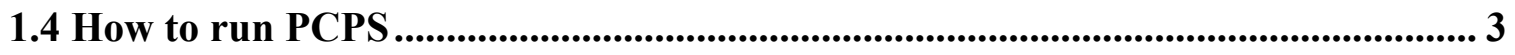

1.5 History and Contact Information....................................................................................... 3

CHAPTER 2: HOW TO NAVIGATE THE PCPS SCREENS ................................. 4

2.1 What are the GUI Pull-Down Menus............................................................... 4

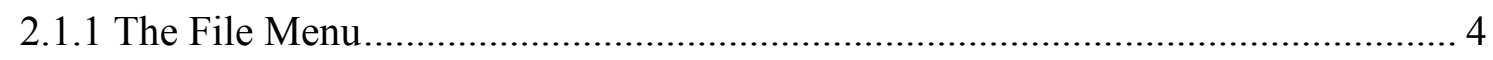

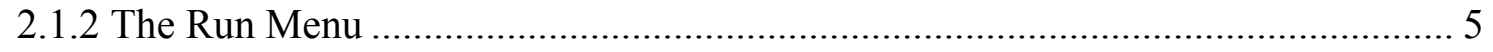

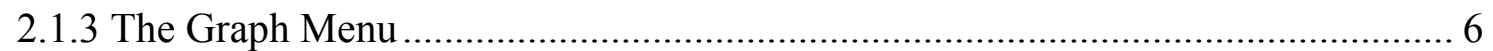

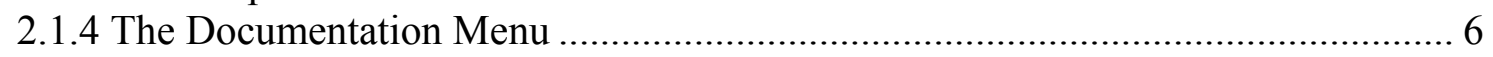

2.2 What are the GUI Input Tabs ........................................................................................ 7

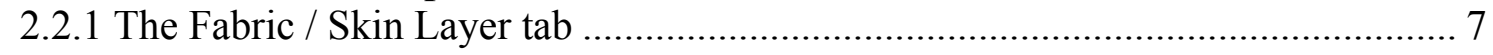

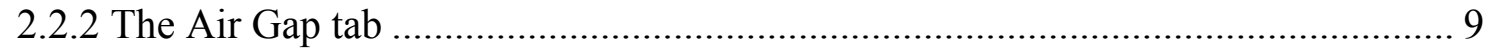

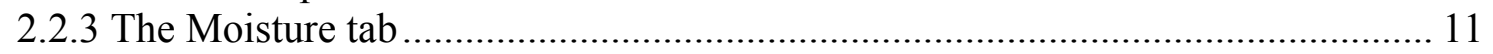

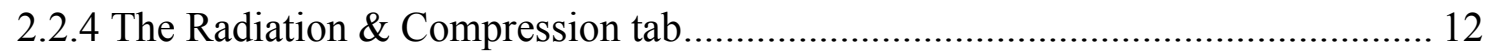

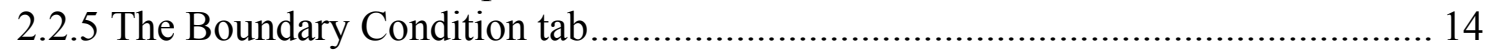

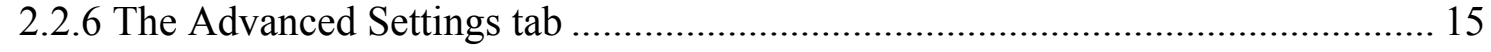

2.3 How to monitor progress through the DOS-Shell..................................................... 16

CHAPTER 3: HOW TO DEFINE AND CONDUCT A SIMULATION.................... 18

3.1 Defining a simulation ........................................................................................ 18

3.1.1 Method 1: Default entry through the GUI ................................................... 18

3.1.2 Method 2: Loading an example through the GUI........................................... 19

3.1.3 Method 3: Importing an external file through the GUI.................................... 19

3.1.4 Method 4: User specified parameters through the GUI tabs............................ 19

3.2 Performing a simulation ........................................................................................... 19

CHAPTER 4: HOW TO ANALYZE THE RESULTS OF A SIMULATION.......... 21

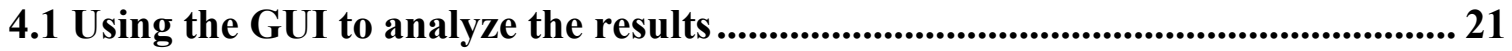


4.1.1 Temperature (front $\&$ back faces) versus Time .............................................. 22

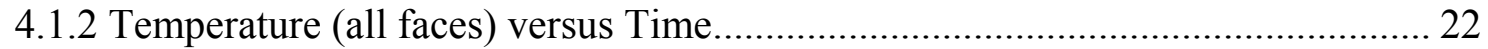

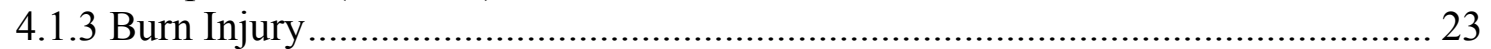

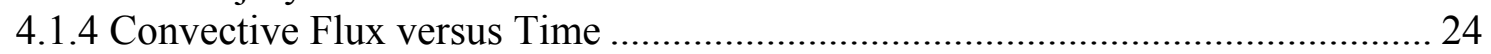

4.1.5 Radiative Flux versus Time ....................................................................... 24

4.1.6 Mass Flux versus Time ............................................................................... 25

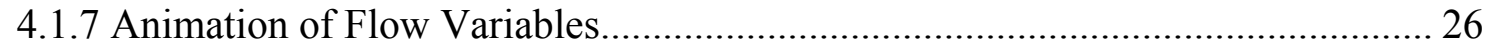

4.1.8 Animation of Terms in Energy Equation........................................................ 26

4.1.9 Animation of Terms in Mass Equation ............................................................. 27

4.2 Using the Output DATA files to analyze the results ............................................... 28

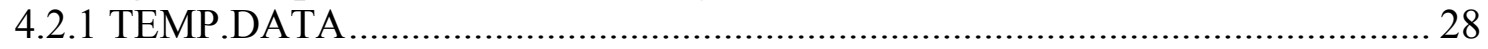

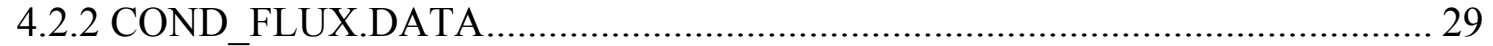

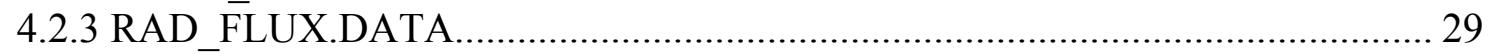

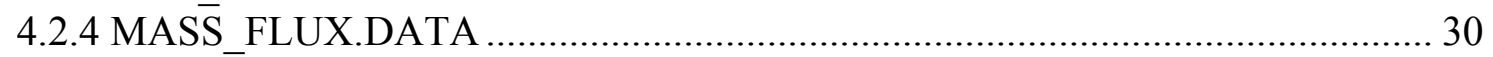

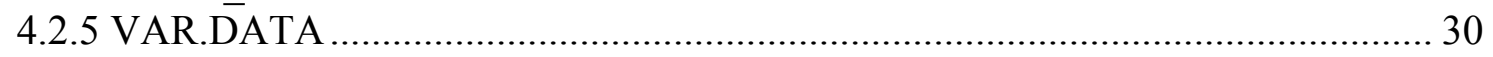

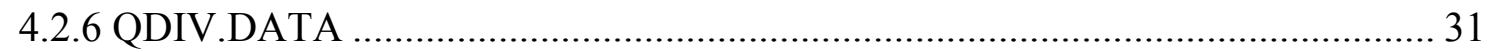

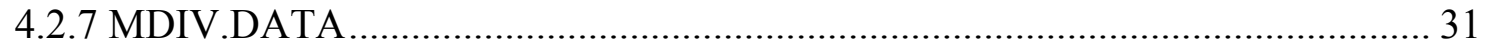

CHAPTER 5: A SAMPLE CASE STUDY USING PCPS ....................................... 33

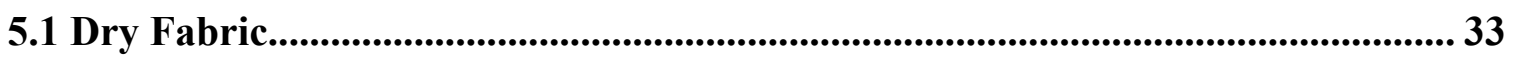

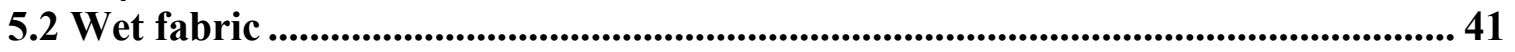

ACKNOWLEDGEMENTS .................................................................................................... 47

APPENDIX A : THE PREDEFINED EXAMPLES ................................................. 48

A.1 Radiative flux definitions.....................................................................................49

A.2 Material, node and temperature definitions.................................................. 49

APPENDIX B : HOW TO CREATE YOUR OWN INPUT FILE............................ 51

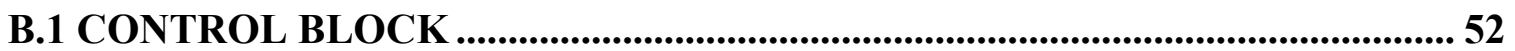

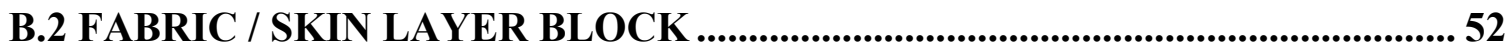

B.3 MOISTURE BLOCK.................................................................................... 53

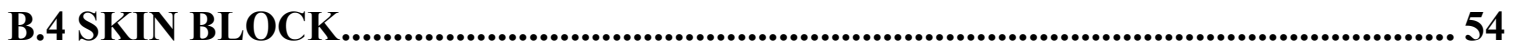

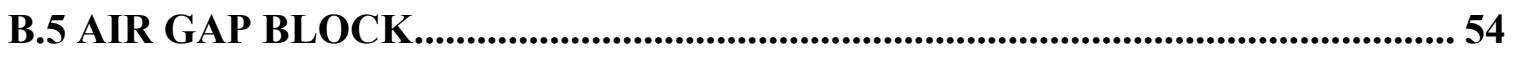

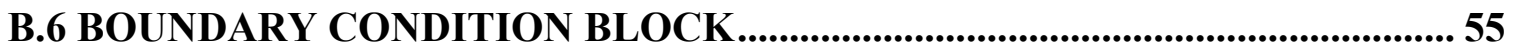

B.7 ADVANCED SETTINGS BLOCK ......................................................... 55 


\section{Disclaimer}

The US Department of Commerce makes no warranty, expressed or implied, to users of the Protective Clothing Performance Simulator (PCPS), and accepts no responsibility for its use. Users of PCPS assume sole responsibility under Federal Law for determining the appropriateness of its use in any particular application; for any conclusions drawn from the results of its use; and for any actions taken or not taken as a result of analyses performed using these tools.

Users are warned that PCPS is intended for use only by those competent in the fields of heat and mass transfer, fire dynamics, thermodynamic and combustion, and is intended only to supplement the informed judgment of the qualified user. The software package is a computer model that may or may not have predictive capability when applied to a specific set of factual circumstances. Lack of accurate predictions by the model could lead to erroneous conclusions with regard to fire safety. An informed user should evaluate all results.

Throughout this document, the mention of commercial software or commercial materials does not imply recommendation or constitute endorsement by NIST, nor does it imply that the products are necessarily those best suited for the intended purpose. 


\section{Chapter 1: Introduction and Installation of PCPS}

\subsection{What is PCPS}

PCPS is an abbreviation for the Protective Clothing Performance Simulator. PCPS is a software tool for predicting the thermal performance of fire fighter clothing, in protecting skin from burn injury that results from exposure to high intensity thermal radiation. PCPS will allow the user to easily set up a numerical experiment using an interactive Graphical User Interface. The interface will allow the user to study transient heat and mass transfer through the multi-layered fabric / skin assemblies with or without air gaps. Fabric layer characteristics, air gap thickness, moisture levels, compression, boundary conditions and other advanced features can be prescribed through this graphical user interface. The results of the simulation can then be interactively visualized using a set of pre-defined $x y$ plots and animations, included with the software.

This manual is intended to provide the basic information necessary to use the PCPS software, to perform a simulation and to interpret the results, but is not intended to develop the rigorous underlying theory behind the numerical simulations. Readers who are interested in these details are referred to the technical manual ${ }^{1}$ of PCPS. The technical manual and this user's manual are included in pdf format with the PCPS software tool.

This manual has been written with the assumption that the user possesses a base knowledge of the Windows ${ }^{\circledR}$ operating system. Specifically, the user should know how to navigate using the Explorer application and be knowledgeable of the basic format of an application window (minimize, maximize and close).

1 K Prasad, W Twilley and J R Lawson, "Thermal Performance of Fire Fighters' Protective Clothing. 1. Numerical Study of Transient Heat and Water Vapor Transfer." NISTIR 6881. August, 2002. 


\subsection{System Requirements}

The software comprising PCPS contains two core components. The first component is a numeric solver written in Fortran 90, precompiled for a specific operating system (file FFPE.exe). The second component of PCPS is a Graphical User Interface written in Java $^{\text {tm }}$ (file GUI.jar). For users performing Windows ${ }^{\circledR}$ installations, a version of the $\mathrm{Java}^{\mathrm{tm}}$ 1.2.2 Runtime Environment is included with the software and is recommended for the best use of the Graphical User Interface. Users wishing to install PCPS on other operating systems, such as UNIX or LINUX, please contact Kuldeep Prasad at the contact location provided below.

The PCPS software requires approximately $2.5 \mathrm{MB}$ of disk storage space for the directly associated applications. Additional disk space is required during a simulation for storage of output file data created during a simulation. PCPS will also require approximately $32 \mathrm{MB}$ of RAM to perform the simulation.

\subsection{How to install PCPS}

To install PCPS, download the file PCPSsetup.exe from the NIST website to the folder in which to PCPS is to be installed. This self-extracting .zip file contains all of the necessary files to run PCPS. Once the file is downloaded, double-click on the PCPSsetup.exe file to begin the installation and unpack the PCPS components. If the computer on which PCPS is installed does not contain the necessary libraries for running $\mathrm{Java}^{\mathrm{tm}}$ files, then run the JREsetup.exe program (Windows ${ }^{\circledR}$ only) contained in the "accessories" subdirectory. This will install the necessary runtime libraries used by the Graphical User Interface.

Once the installation is complete and PCPS is running successfully, the self-extracting file as well as the accessories subdirectory can be deleted. Be aware that the installation program for PCPS will make no changes to registry with regard to the path or any environment variables. The user can therefore move or copy the executable portion of the software at a later time to any desired folder. If doing this, all of the following files must be moved or copied to the directory from which it is desired to run PCPS,

PCPS.bat

GUI.jar

FFPE.exe

sat.data

The following folders should also be moved or copied, documentation

examples

The "documentation" folder contains the User's Guide and the Technical manual. The "examples" folder contains specific files for example simulations that are accessible from within PCPS. 


\subsection{How to run PCPS}

To start PCPS, double-click on the icon marked PCPS.bat (or simply PCPS if file extensions are turned off) in a Windows ${ }^{\circledR}$ Explorer window. This action should open three windows: a DOS shell, providing information about the progress of a simulation; a Graphical User Interface for controlling the simulation, entering data and viewing results; and a "Getting Started" window providing a simplified set of instructions for running PCPS. The "Getting Started" window may be closed at any time and then reopened by selecting "Getting Started" from the Documentation pull-down menu. The other two windows (the DOS shell and the Graphical User Interface) must remain open at all times. Closing either window will terminate PCPS.

Chapter2 describes how to use the Graphical User Interface pull down menus and the various input tabs for entering input data. Four different methods for prescribing input data and for defining a simulation are presented in Chapter 3. The focus of Chapter 4 is on the analysis of the results of a typical simulation and on the structure of the output data files for post-processing. A sample case study for using the software tool for studying dry and wet fabrics has been presented in Chapter 5.

\subsection{History and Contact Information}

The beta version of PCPS was completed on August 15, 2002. The Building and Fire Research Laboratory of the National Institute of Standards and Technology (NIST) developed the Fortran 90 program. The GUI was developed under the guidance of Kuldeep Prasad and implemented by Hoang Minh Ho Dac of the University of California, Irvine as part of the Summer Undergraduate Research Fellowship (SURF) 2002 program. Please direct all inquiries and comments about this software to:

Dr. Kuldeep Prasad

Polymers Building (224), Room B258

National Institute of Standards and Technology (NIST)

100 Bureau Drive, Mail Stop 8663

Gaithersburg, MD 20899-8663

Tel: (301) 975-3968

Fax: (301) 975-4052

Email: kuldeep.prasad@nist.gov 


\section{Chapter 2: How to navigate the PCPS screens}

The primary means of interacting with PCPS is through the Graphical User Interface (hereafter referred to as the GUI), shown in Figure 2.1, and the DOS shell status window, shown in Figure 2.2. The GUI has two basic elements, a series of pull-down menus near the top of the window and a set of input tabs comprising the majority of the window. Through these elements, the user enters material and fire parameter data, starts or stops a simulation, post-processes the results, and obtains help. The DOS shell displays the status of a simulation being conducted.

\subsection{What are the GUI Pull-Down Menus}

The pull-down menus are used for file manipulation, for controlling the execution of a simulation, for graphically visualizing the results of a simulation and for accessing the documentation for PCPS. The menus and available tasks under each menu are described below.

\subsubsection{The File Menu}

The file menu is a list of tasks related to file manipulation in PCPS. The sub-menu task items are,

New - Begin a new simulation, clearing all data from the input tabs.

Import Examples - Load an example input file from a pull down menu. Several example input files have been provided with the PCPS software. These example input files cover a wide range of fabric/skin configurations, degree of external radiative flux and dry or wet fabric layers. Clicking "Import Examples" will open a sub-menu listing of input examples that are provided for the user. The possible scenarios as well as the characteristics of these scenarios can be found in Appendix A.

Import Input File - Load conditions from an external (user created) file. 
Save \& Print Input - Open the INPUT file that resides in the directory from where PCPS was executed and display it on the screen. The file can also be printed or saved to a different name or location using this menu item.

Save \& Print Output - Open a specified output file that resides in the directory from where PCPS was executed and display it on the screen. The displayed file can also be printed or saved to a different name or location using this menu item.

Exit - Close all windows and quit PCPS.

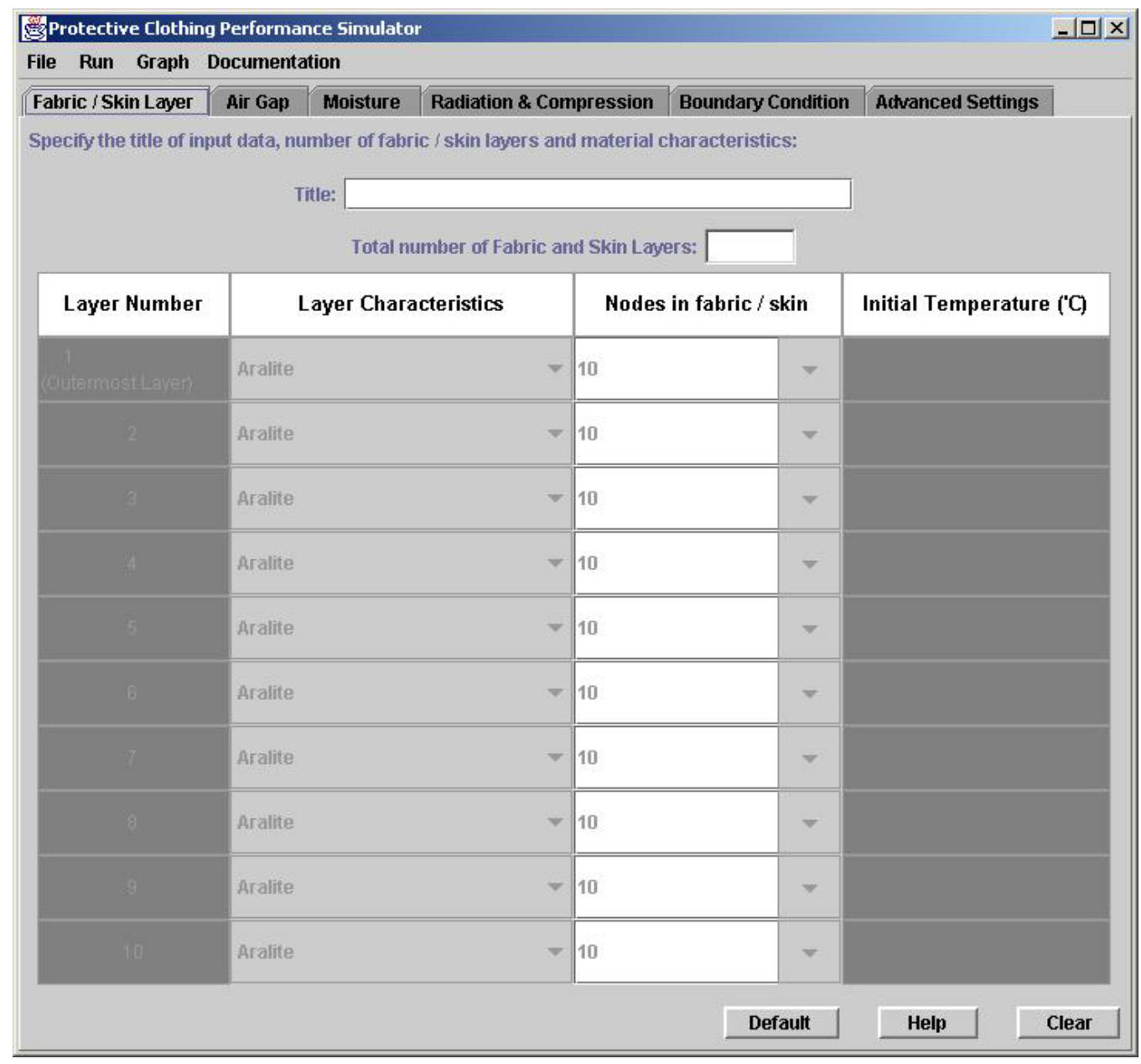

Figure 2.1: The Graphical User Interface (GUI)

\subsubsection{The Run Menu}

The run menu controls a simulation and contains two tasks, 
Execute - Begin the numerical simulation using the input parameters specified in the Graphical User Interface. The input parameters specified in the GUI will be checked for consistency and completeness and an INPUT file will be created in the working directory.

Stop - Interrupt the numerical simulation that was initiated using the Execute command.

\subsubsection{The Graph Menu}

The graph menu contains a list of graphs available for processing the results of a simulation. These graphs show the progress of some key variables as a function of space and time. When selected, a graph is displayed in its own window. Chapter 4 gives detailed instructions on how to analyze the results of a simulation.

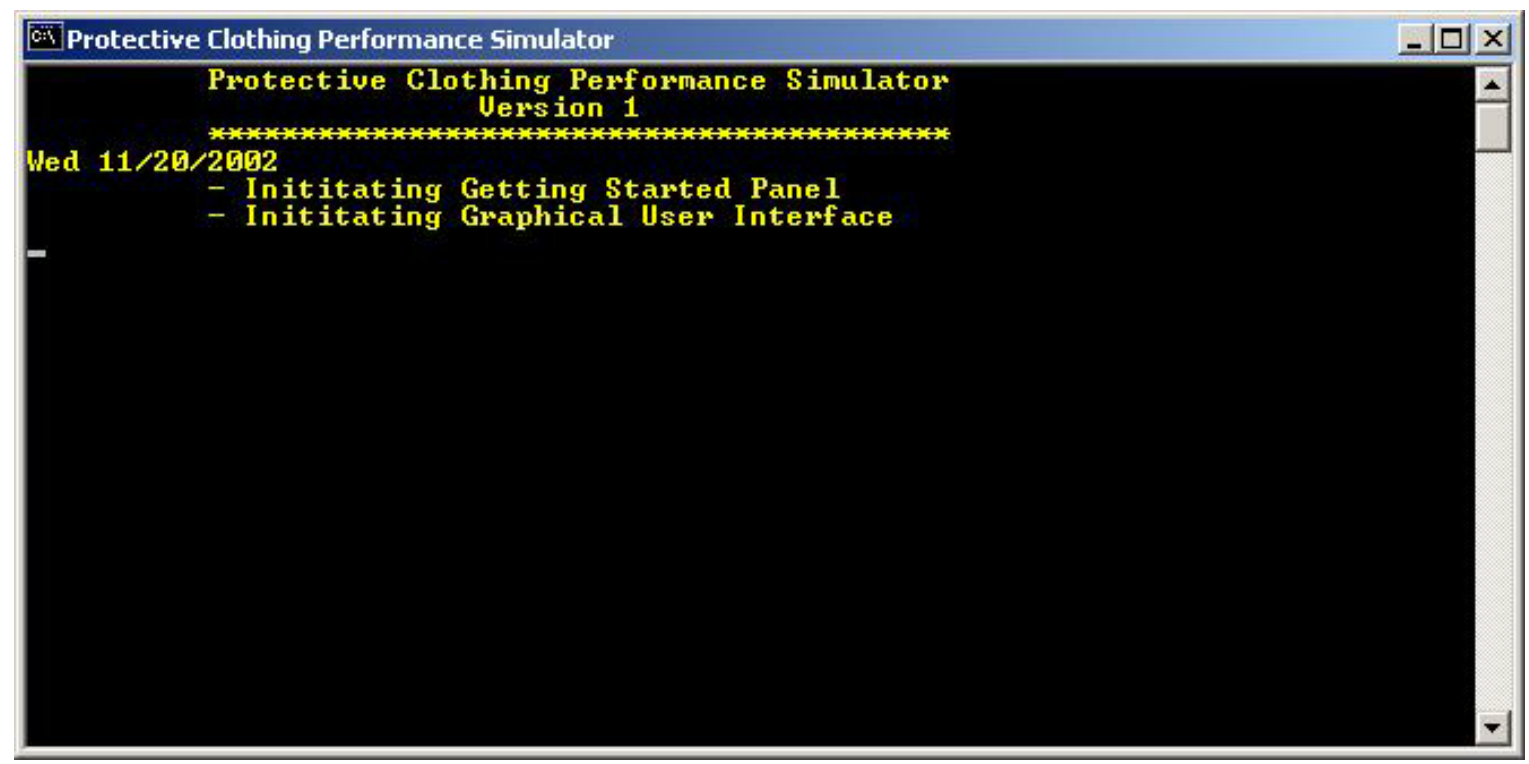

Figure 2.2: The DOS shell window

\subsubsection{The Documentation Menu}

The "Documentation" menu contains a list of documentation available from within PCPS. The User and Technical manuals are in PDF format, (an appropriate viewer must be available). The other options will open their own display window. The documents available from this menu are,

Getting Started - A simplified set of instructions for running PCPS. This is the third window opened whenever PCPS is started.

User's Manual - Thermal Performance of Fire Fighters Protective Clothing. 2) Protective Clothing Performance Simulator - User's Manual. "Kuldeep Prasad, Hoang Minh Ho Dac, Scott Kukuck”, NISTIR 6901, 2002. This manual is provided in pdf format. 
Technical Manual - Thermal Performance of Fire Fighters Protective Clothing. 1) Numerical Study of Transient Heat and Water Vapor Transfer, "Kuldeep Prasad, William Twilley, J. Randall Lawson”, NISTIR 6881, 2002. This manual contains the underlying theory and equations utilized by the solver. The manual is provided in pdf format.

Unit Conversion - A list of common conversion formulas. The parameters, which must be supplied for PCPS, are required to be in specific units. These formulas aid in any necessary calculations.

About PCPS - Version and contact information for PCPS.

\subsection{What are the GUI Input Tabs}

The material specifications and other simulation parameters are entered through a series of six input tabs located beneath the pull-down menus. Here the characteristics of the fabric layers, air gaps, moisture distribution, radiative flux, initial and boundary conditions and advanced features are all recorded. Each tab corresponds to specific feature(s) available for PCPS and is covered in the following sections.

At the bottom right of each tab is a set of three buttons. The "Default" button inserts a default set of conditions into the tab. The "Clear" button erases any information already entered on the tab. Both options should be used with care as some information supplied on the given tab has a direct influence on subsequent tabs.

A "Help" button has been provided at the bottom right hand corner of each tab. Clicking this button, opens an additional window that provides a simplified list of instructions and suggestions for inserting input data. These help windows can be closed when not in use and then reopened at the user's discretion.

\subsubsection{The Fabric / Skin Layer tab}

The Fabric / Skin Layer tab (See Figure 2.3) provides information regarding individual layers of a multi-layered fabric. Located at the top-center of this tab is space for entering the title of the simulation. The title is then displayed on all graphs and is listed in each output data file.

Immediately below the title is a spot to specify the number of fabric layers to be included in the simulation. PCPS allows for up to 10 separate layers for a given simulation. Once a number is entered in this location, the appropriate number of fabric layers become active. This field also has a direct influence on the Air Gap and Moisture tabs. The appropriate sections of these tabs also become (in)active once a quantity for the number of fabric layers is entered. 
For each layer, the user must specify the material characteristics (fabric or skin layer), the number of nodes to be distributed throughout the material, and the initial temperature of the material (in degrees Celsius). The numerical ordering of the layers progresses from left to right with incident radiation striking the left-most layer first (i.e. for three layers, layer one is the far left layer upon which the radiation is incident, layer two is the middle layer, and layer three is the far right layer furthest from the radiation source).

The material is specified from a drop-down list of possible choices. The currently available choices are,

1. Aralite $^{\circledR}$

2. Breathe-Tex ${ }^{\circledR}$

3. Breathe-Tex ${ }^{\circledR}$ Plus

4. Cotton Duck

5. Nomex ${ }^{\circledR}$ E-89 Crosstech $^{\circledR}$

6. Neo-Guard ${ }^{\circledR}$

7. Nomex ${ }^{\circledR}$ III-Defender ${ }^{\mathrm{TM}}$

8. Nomex ${ }^{\circledR}$ IIIA Pajama Check Crosstech ${ }^{\circledR}$

9. PBI $^{\mathrm{TM}}$ Kevlar $^{\circledR}$ Kombat $^{\mathrm{TM}}$

10. Scotchlite ${ }^{\circledR}$

11. Epidermis

12. Dermis

13. Subcutaneous Tissue

14. Kevlar ${ }^{\circledR}$ Batting

15. Aramid

Be aware that skin modeling requires the use of all three sub-layers of skin (epidermis, dermis and subcutaneous tissue) as individual material layers. 


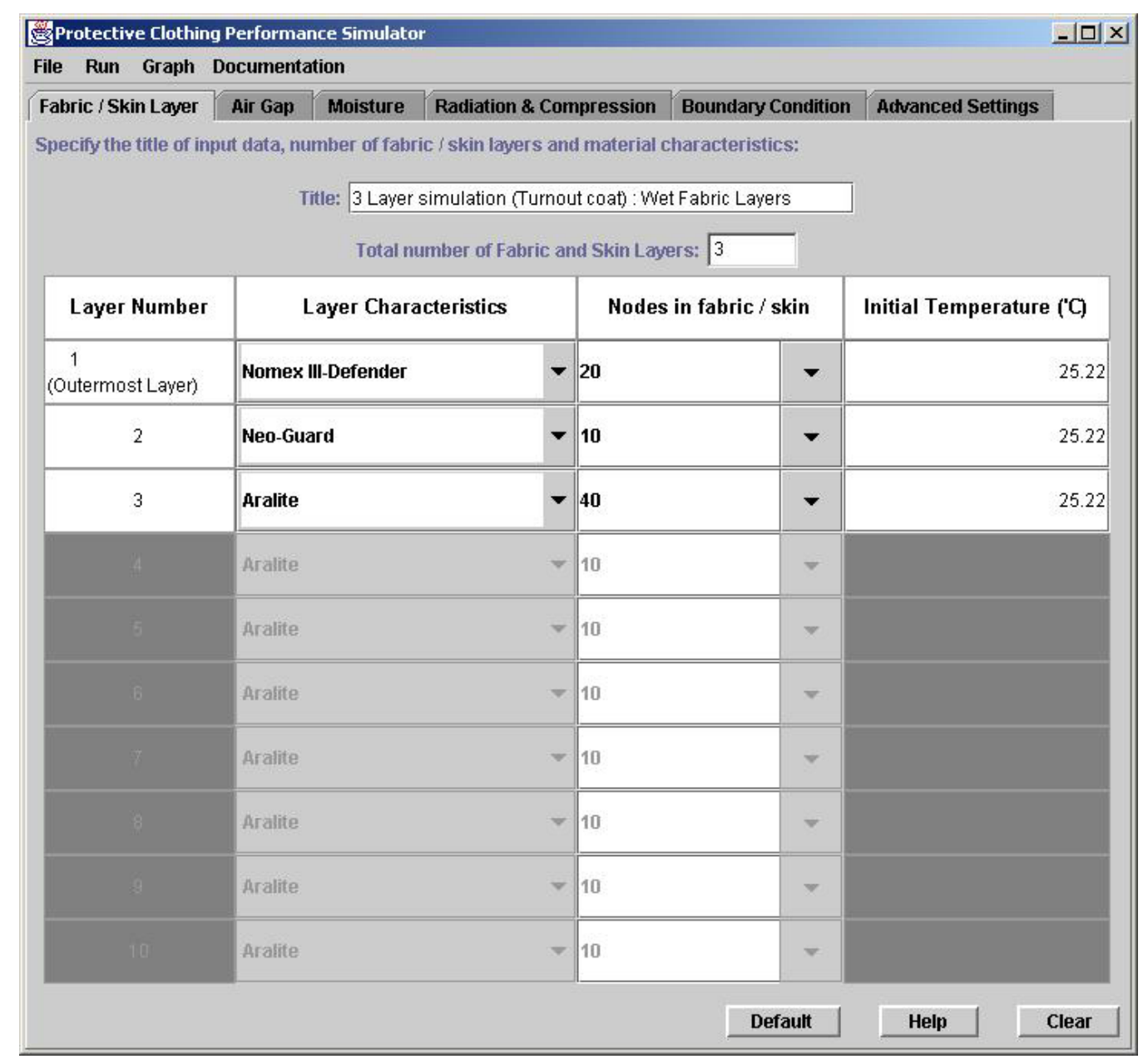

Figure 2.3: The Fabric / Skin Layer Tab

The user also specifies the number of nodes that will be used to resolve the calculation of heat and mass transfer through the fabric / skin layers. This is accomplished by entering the number of nodes either manually or from the available drop-down list. The number of nodes used can have an impact on the accuracy of the simulation as well as on the CPU cost. The accuracy of the calculation improves as the number of nodes increases. If the number of nodes is too small, the simulation may become unstable or the solution may exhibit spurious oscillations. Under such conditions, the DOS shell window may display an error message such as "Time step too small" or "Time step is negative." This problem can be corrected by increasing the number of nodes, especially in regions where the convective and radiative fluxes are very high, or the rate of vaporization is expected to be a very large number. In general, the accuracy and stability of the calculation will improve as the number of nodes increases. However the computational cost of the calculation will also increase as the number of nodes increases.

\subsubsection{The Air Gap tab}




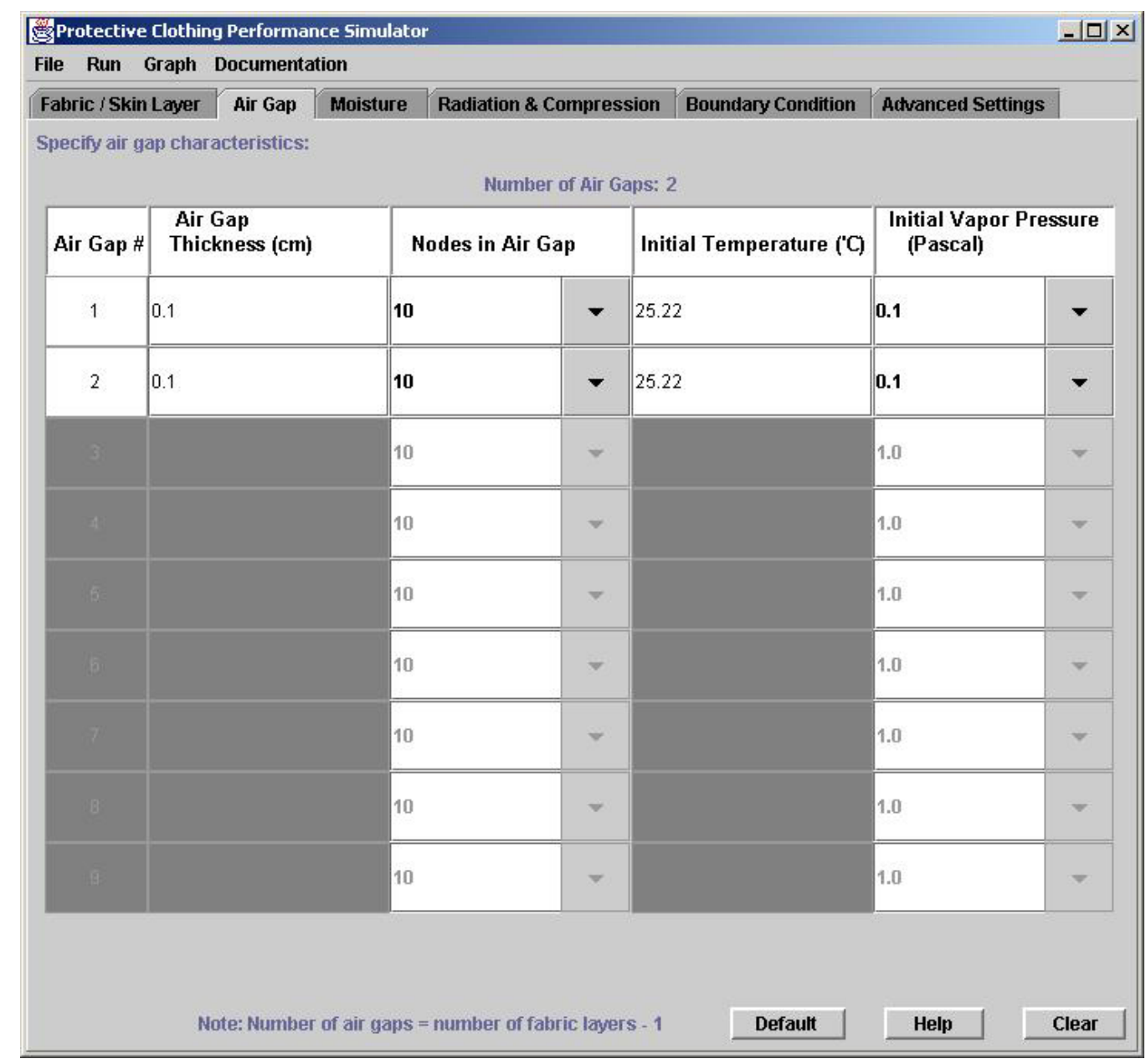

Figure 2.4: The Air Gap Tab

The air gap tab (Figure 2.4) is used to specify the characteristics of the air gaps that separate the fabric layers. The total number of possible air gaps is equal to the number of layers of fabric / skin minus one. The appropriate number of possible air gaps becomes active on this tab once the number of fabric / skin layers has been entered on the Fabric / Skin Layer tab. Gaps are numbered consecutively from left to right similar to the material layers (i.e. air gap number one corresponds to the gap between material layers one and two, air gap number two corresponds to the gap between material layers two and three, etc.).

For each air gap, the user must specify the thickness, number of nodes, initial temperature and initial vapor pressure. The thickness (in centimeters) and the initial temperature (in degree Celsius) are entered into the corresponding areas of the tab while the number of nodes and the initial vapor pressure (in Pascal) are either entered manually or selected from a drop-down menu. If no air gap is desired between the layers of fabric / skin, the number of nodes for the corresponding gap should be set to zero. As for the material layers, the greater the number of nodes the more accurate the solution, but the longer the time required to complete the simulation. The initial vapor pressure is the partial pressure 
of water vapor and should be less than or equal to the saturation pressure. Vapor pressure is a function of the temperature and the relative humidity.

\subsubsection{The Moisture tab}

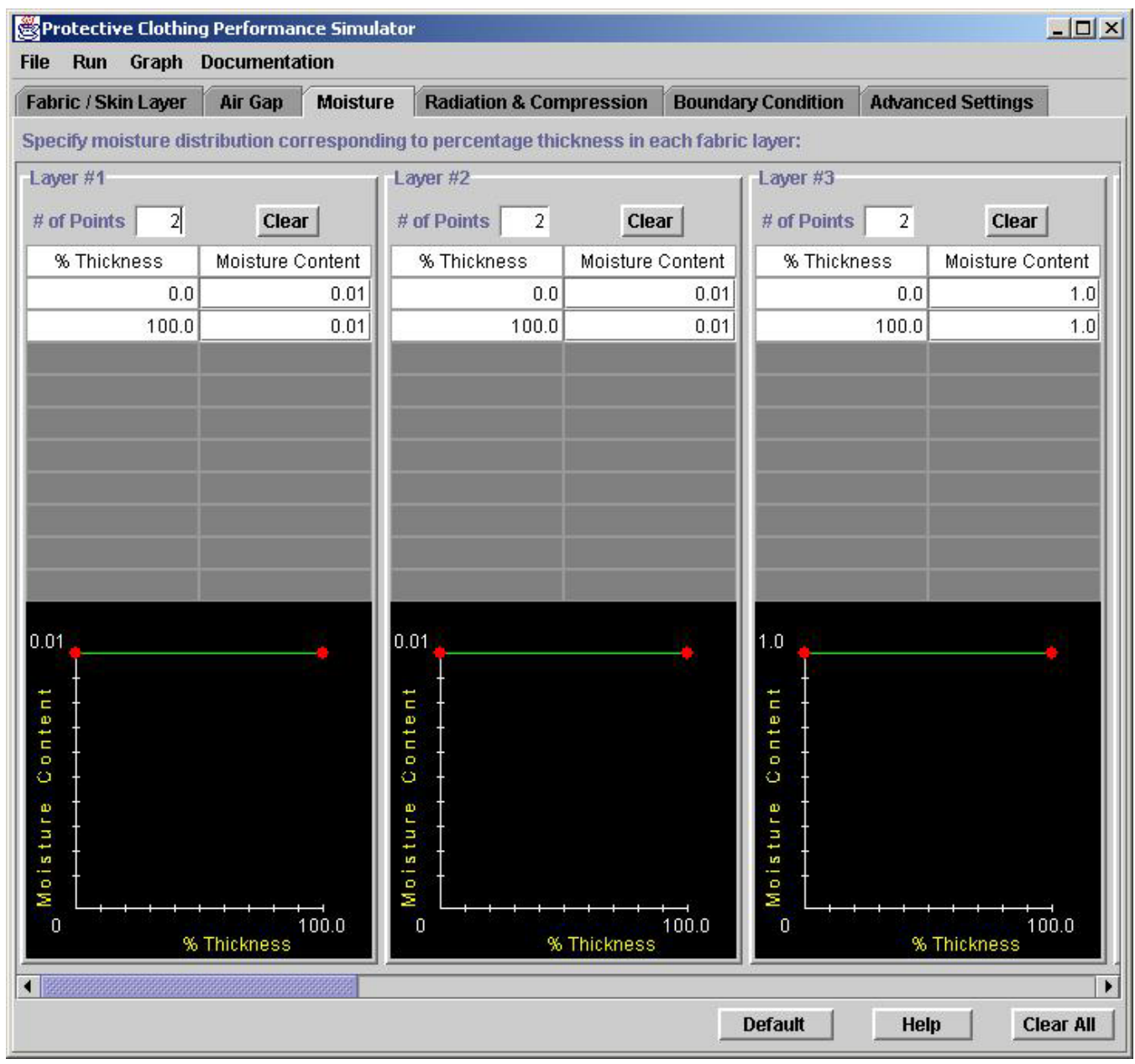

Figure 2.5: The Moisture Tab

The moisture tab (Figure 2.5) is used to specify an initial distribution of water trapped within the fabric layers and thus allows for the possibility of wet fabrics. This is accomplished by defining the moisture content as a function of thickness of the fabric. The moisture content is a non-dimensional number and is equal to the density of moisture divided by the density of dry fabric. Thus a moisture content value of 1 would indicate that the density of moisture in the fabric is locally equal to the dry density of the fabric itself. Heavily soaked fabrics can have moisture content of 5-10, depending on the fabric, while fabrics subjected to moist air may have very small moisture content value of 0.1 , again depending on the fabric characteristics and temperature. 
The moisture distribution is described as a piecewise linear function of the percentage of fabric thickness. For each layer, the user must first specify the number (\#) of points that will be used to specify the moisture distribution. Notice that if the number of points is 3 , then three lines will be highlighted. More points (up to a maximum of 10) can be used to specify a very complex moisture distribution pattern. Under \% thickness, enter the location where the moisture content is specified by entering a number between 0 and 100 (since these are \% thickness). A graphical representation is displayed under the table of values to aid the user in defining the moisture content. At any time pushing the "Clear" button for a specific layer will clear the data for that layer.

\subsubsection{The Radiation \& Compression tab}

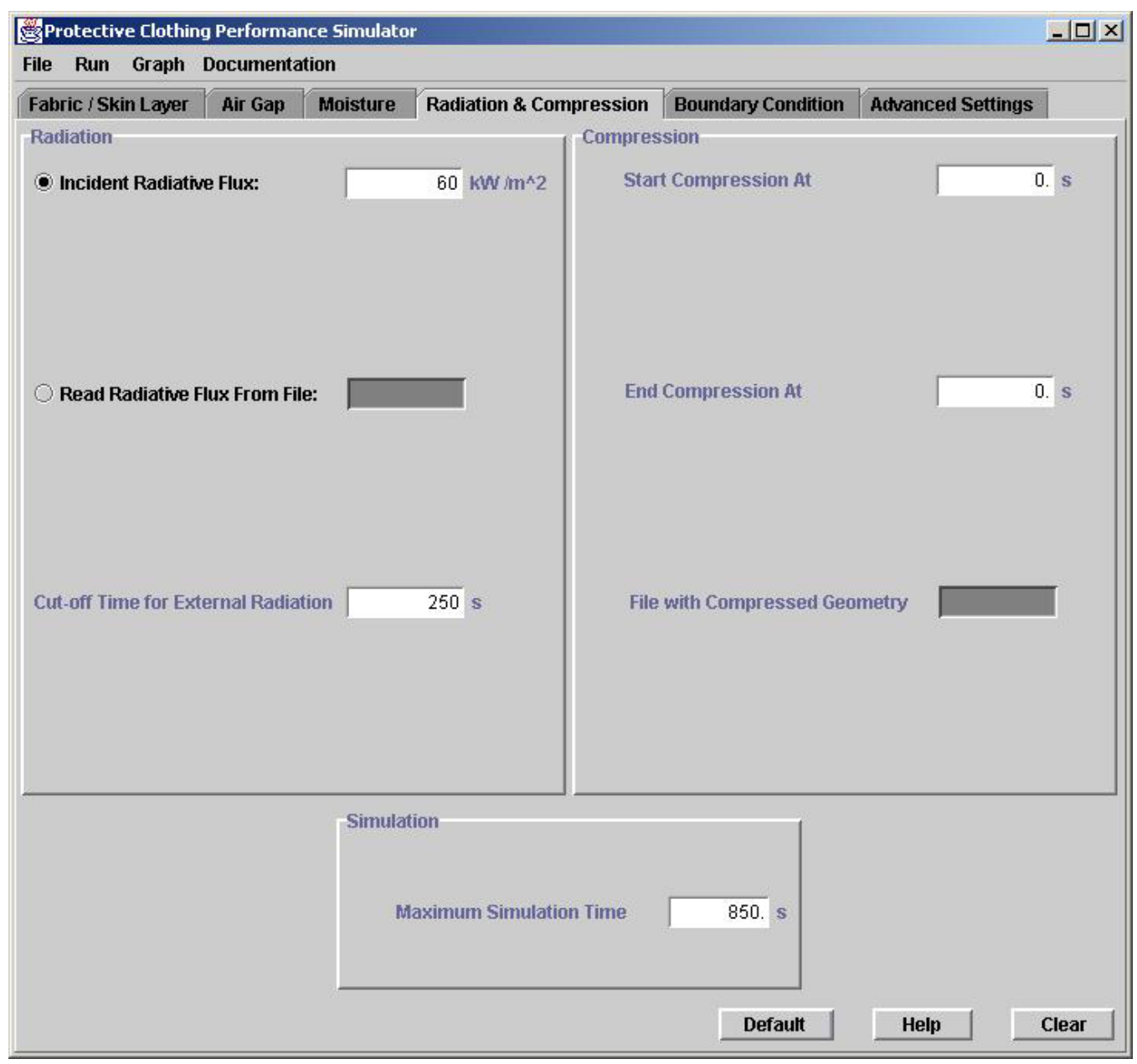

Figure 2.6: The Radiation \& Compression Tab

The Radiation \& Compression tab (Figure 2.6) is used to specify the incident radiation upon the left boundary of the first fabric or skin layer. Specifying the incident radiative flux can be done using either of two methods. The first method subjects the fabric to a constant flux and is implemented by selecting the top radio button "Incident Radiative 
Flux." A radiative flux value in units of $\mathrm{kW} / \mathrm{m}^{2}$ is then entered in the adjoining box. The second method allows for time varying heat flux and is implemented by selecting the middle radio button "Read Radiative Flux From File." Data is imported to the Fortran solver through an external file, the name of which is specified in the corresponding box. The fixed format file (described below) must reside in the same directory from which PCPS is being run.

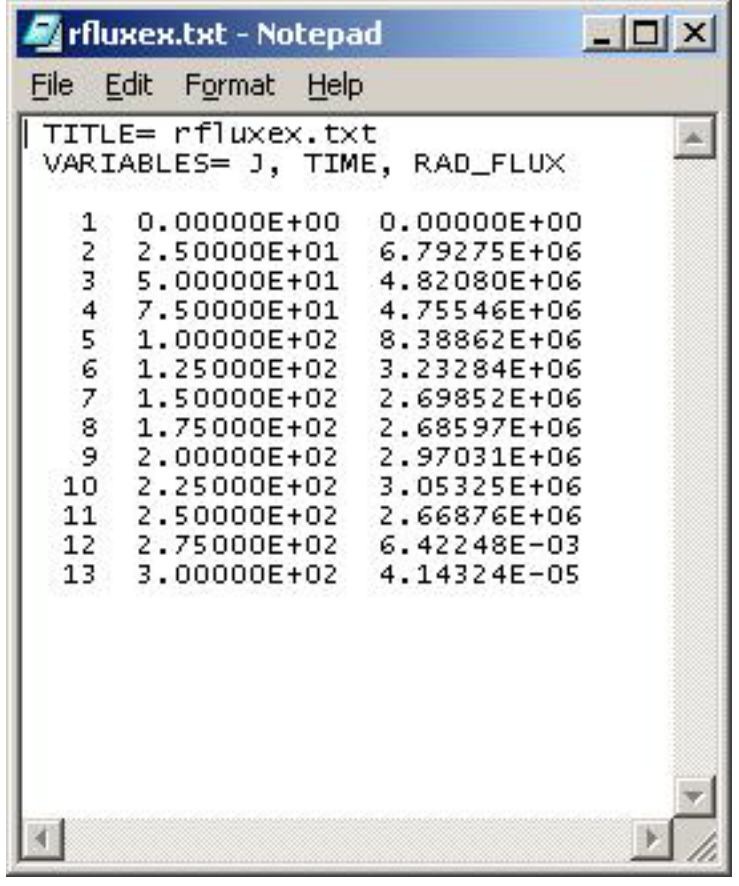

Figure 2.7: Sample radiative flux file

Shown in Figure 2.7 is an example file used to specify a time varying radiative flux. The first three lines of the file are dummy lines not used by PCPS and are a good location to put identifying information for the file. The radiative flux data begins on the third line and is of the form,

\section{IIIIbbT.TTTTTE+00bbF.FFFFFE+00b}

The letters IIII denote a 4-digit integer value. This number is not utilized by PCPS and so it is used in the example as a counter. The letters T.TTTTTE +00 denote the time (in seconds) of the data point in exponential form. The letters F.FFFFFE +00 describe the radiative flux in units of erg $/ \mathrm{s}-\mathrm{cm}^{2}\left(1 \mathrm{erg} / \mathrm{s}-\mathrm{cm}^{2}=10^{-6} \mathrm{~kW} / \mathrm{m}^{2}\right)$ also in exponential form. The ' $b$ ' value represents a blank space. For those familiar with Fortran programming, this line format as ' $\mathrm{I} 4,1 \mathrm{x}, 2(\mathrm{E} 12.5,1 \mathrm{x})$.'

Two time values are supplied on this tab. The first, "Cut-off Time for External Radiation," determines when the fabric is no longer subjected to the radiative flux. The fabric is then allowed to cool by either convective or conductive means. The second 
time, the "Maximum Simulation Time," determines the point at which the simulation is to be stopped. PCPS incorporates a skin damage model to assess thermal injury (when skin layers are included). Due to the finite time required for the fabric to cool, thermal injury is possible even after the radiative flux is stopped, thus the maximum simulation time may be larger than the radiation cut-off time.

\subsubsection{The Boundary Condition tab}

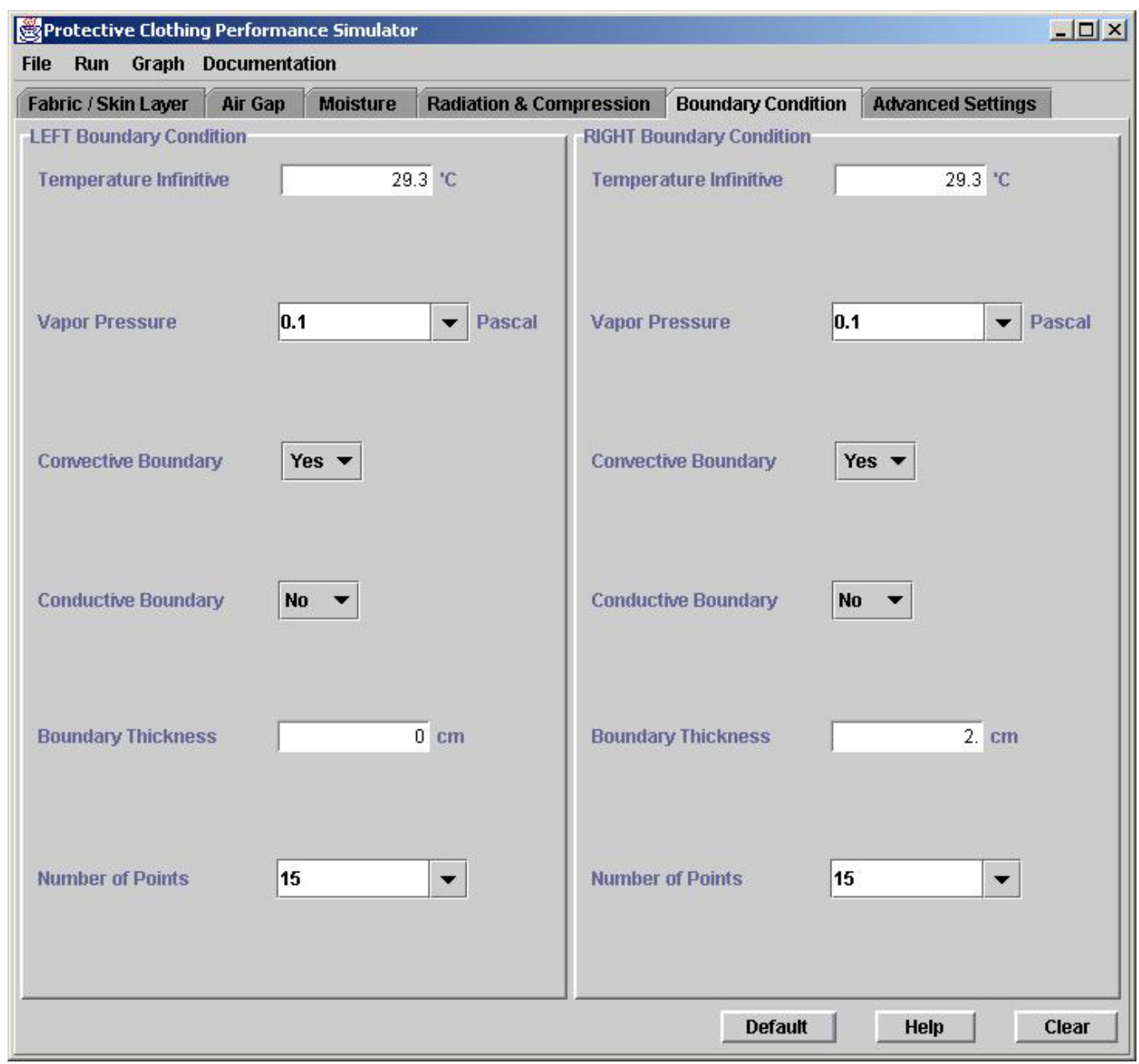

Figure 2.8: The Boundary Condition Tab

The Boundary Condition tab (Figure 2.8) supplies the necessary boundary conditions (in addition to the already specified radiative flux) to be utilized for the simulation. One of two possible boundary conditions can be specified for a boundary, either convective heat transfer or conduction. Both conditions use the information entered in the first two boxes of the tab. For convective conditions, the "Temperature Infinitive" and the "Vapor Pressure" are the conditions assumed in a far field ambient. For conduction, these are fixed values at the far end of a conductive layer. 
When convective boundary conditions are used, natural convection correlations determine the heat transferred from the boundary to an air ambient. This transfer is a function of the temperature of the boundary as well as a characteristic length of the material (entered on the Advanced Settings tab).

For conductive boundary conditions, an additional layer is utilized that supplements the system. The layer is assumed to be composed of air through which only conductive transport occurs. No convective correlations are included. The thickness and nodal resolution of the boundary layer are required inputs for conductive boundary conditions. Be aware that although the boundary thickness and nodal resolution is not used for convective conditions, a non-zero value is required although it is not used in the calculations.

\subsubsection{The Advanced Settings tab}

The advanced settings tab (Figure 2.9) is for optional conditions not included on the previous tabs. The first two options on the left pertain to skin modeling. When the three sub-layers of skin are included as material layers, the skin model option should be set to true. This incorporates effects and boundary conditions that are appropriate for the body (as opposed to convection or conduction in air). Any condition specified for the right boundary in the Boundary Condition tab is subsequently ignored. A core body temperature is required. When skin sub-layers are not included, the skin model option must be set to false. A core body temperature value must still be present although it is not used in the calculations.

The third item on the left of the tab sets an initial time step to be used for the calculation. As a simulations proceeds, PCPS determines the appropriate next time step however, an initial step must be supplied.

The first two options on the right side of the tab supply information regarding convective boundary conditions for the fabric. The characteristic length used in the correlation is entered and should in some way be representative of the size of the material. The orientation is specified from the appropriate setting in the drop-down box. For horizontal orientations, the fire is assumed incident from above the material. The left layer is thus considered the top layer with progression to the right corresponding to progression downward.

The third item on the right describes the nature of the coefficients (diffusivity, conductivity, etc.) used in the governing equations. Many of these coefficients exhibit some dependence on the temperature, especially over the range of temperatures observed in fires. The transport property drop-down menu informs the solver whether to use temperature dependent properties or constant values for these coefficients. The default (and recommended) setting is to use temperature dependent properties.

The final item, Thermocouple Output, causes the creation of an additional output file entitled, "thrmcpl.data." This file is not used in any of the graphs contained within PCPS but is a more detailed temperature listing for the left and right boundaries of the fabric. 


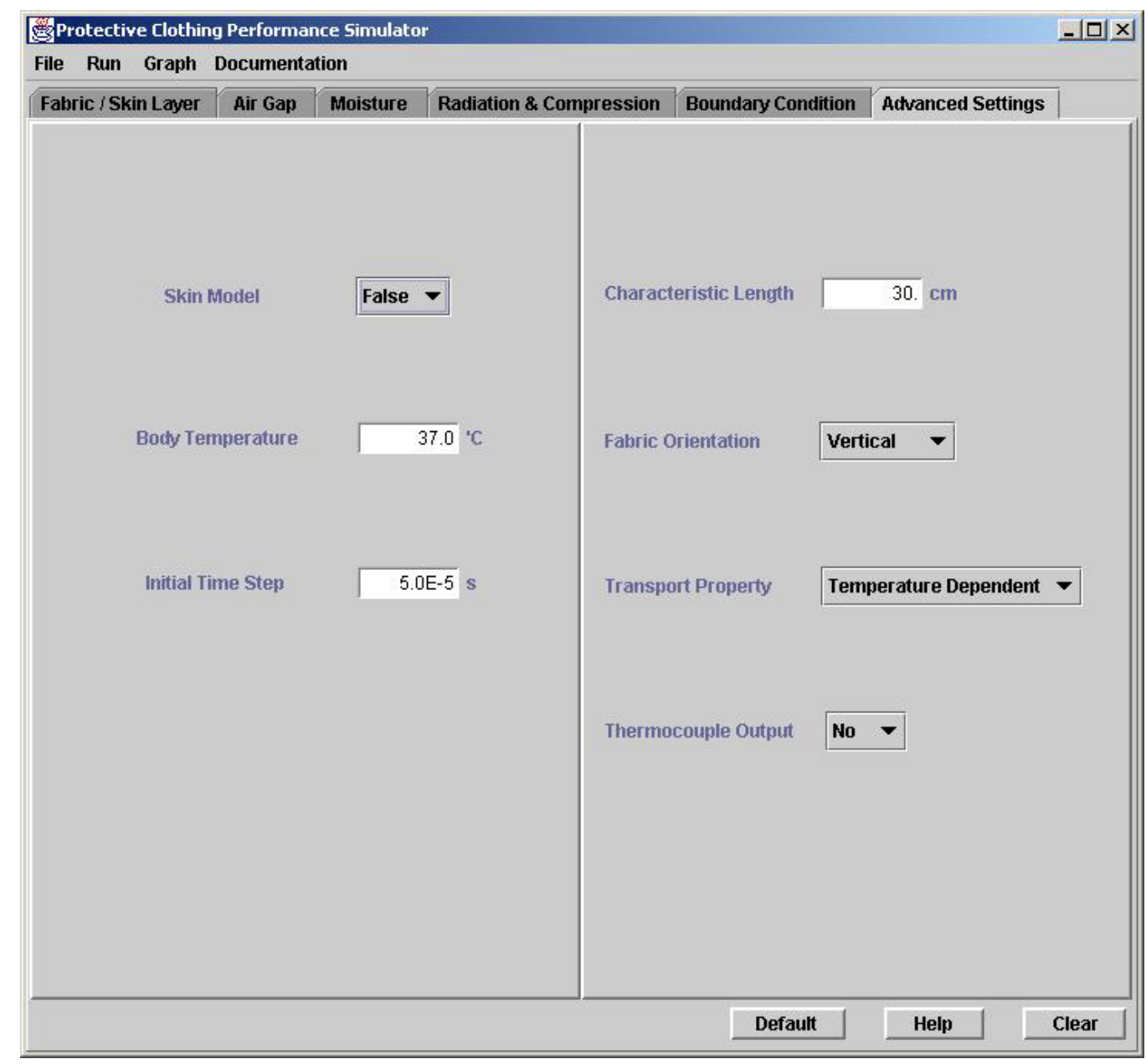

Figure 2.9: The Advanced Settings Tab

\subsection{How to monitor progress through the DOS-Shell}

The DOS shell window, opened when PCPS is started, provides active feedback to the user regarding the progress of a simulation. When first opened the window displays version information for PCPS and the date, see Figure 2.2. As execution starts data is printed to this window at specific intervals such as shown in Figure 2.10. Following a successful simulation, the window displays simply the phrase, "FFPC Analysis: finished.". The time displayed corresponds to the last data point and the desired run time of the simulation. An example of a successful completion is included in Figure 2.10.

If for any reason a simulation fails, diagnostic messages will be printed to the DOS shell window. Using this information the user can correct any mistakes and then restart the simulation. 


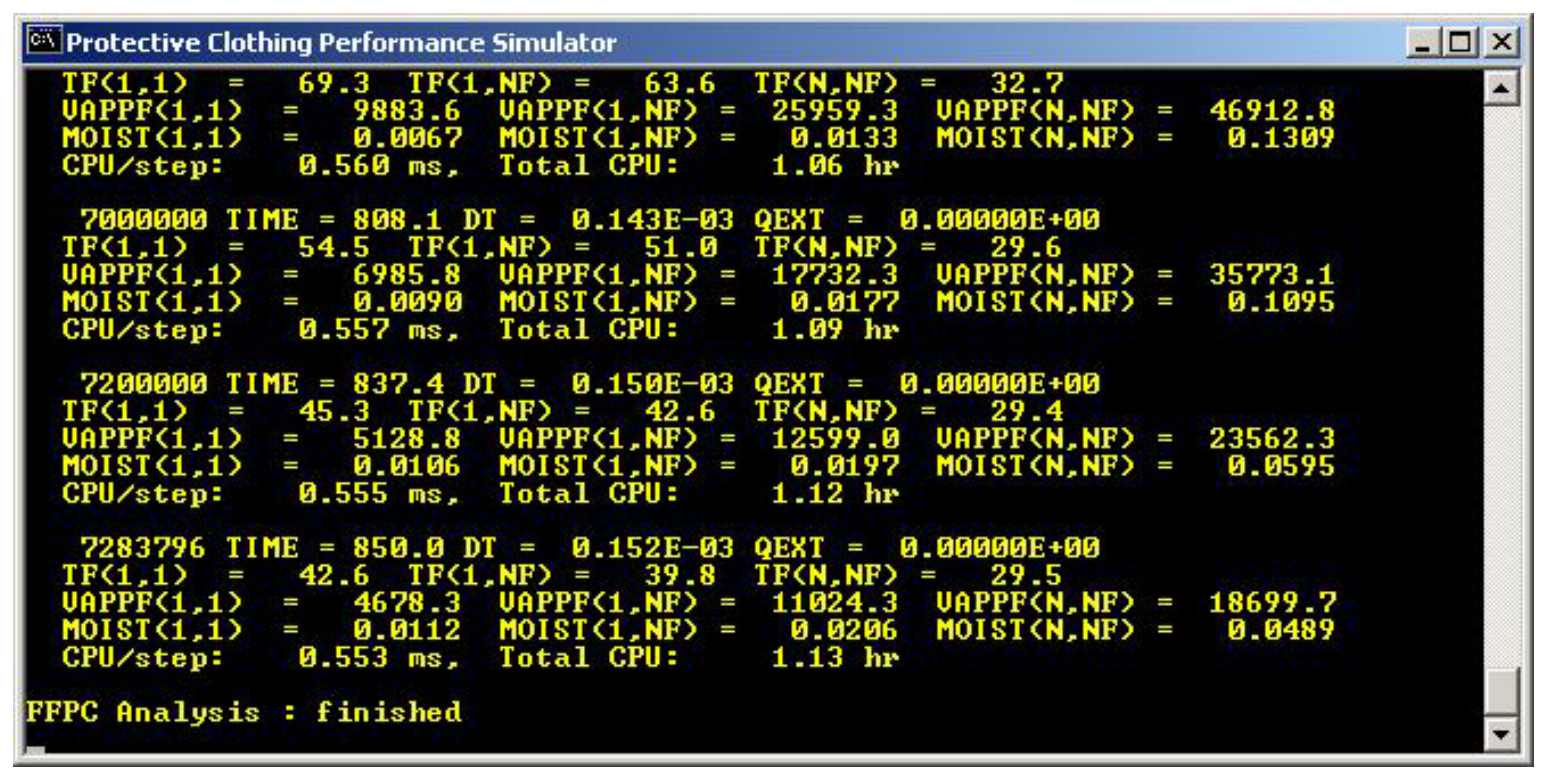

Figure 2.10: DOS shell for successful simulation 


\section{Chapter 3: How to define and conduct a simulation}

The parameters used to define a simulation for PCPS are entered on the various tabs of the Graphical User Interface. A translator then takes the information from the input tabs when a simulation is started (Execute under the Run pull-down menu) and stores the information in an external ASCII text file entitled INPUT (in the required format). This file is located in the directory from which PCPS is being run. The Fortran solver then reads this information and performs the requested calculations.

The INPUT file is created only when a simulation is executed and thus the information on the tabs is not placed within an INPUT file until this action is taken. As a result the presence of data on any input tab does not imply that an INPUT file exists. Moreover, information contained in an already present INPUT file is overwritten when the next simulation is executed from the GUI. It is therefore recommended that following a successful simulation the INPUT and associated output files are saved to a different name / location. The INPUT file (when it exists) can be viewed by selecting Print \& Save Input from the File pull-down menu. This opens a separate display from which it is possible to print, save or close the file.

\subsection{Defining a simulation}

PCPS provides four methods to supply information for the INPUT file. A combination of these methods can provide a powerful way of systematically defining and conducting analyses.

\subsubsection{Method 1: Default entry through the GUI}

The easiest method of supplying information to the GUI and creating an INPUT file is to utilize the "Default" button available on each of the GUI input tabs. The user should visit each of the six tabs and activate the default button at the bottom of each tab. When this button is pressed, a default set of information is entered on each tab. The user can then go to the "Run" menu and click Execute to start a numerical simulation. The corresponding INPUT file and OUTPUT data files can also be viewed through the "File" menu. 


\subsubsection{Method 2: Loading an example through the GUI}

Provided within PCPS are a series of example simulations that can be accessed from Import Examples in the "File" pull-down menu. The information for these simulations is contained in a series of files stored in the "examples" subdirectory. By selecting a specific case, the information contained within these files is loaded into the input tabs of the GUI. Once a simulation is begun, an INPUT file reflecting these conditions is created.

\subsubsection{Method 3: Importing an external file through the GUI}

It is also possible to import a complete set of conditions from a user-specified external file. This done by selecting the Import Input File option from the "File" pull-down menu. A dialog window opens through which the user identifies the specific file to import.

The file to import is a complete INPUT file and must be in the correct format. The rules for creating this file are provided in Appendix B. This file can be created in a wide range of applications, however it must be in an ASCII (plain text) format. If using a word processor to create the file, the user must be careful not to introduce any formatting to the file, as this will cause the import to fail.

\subsubsection{Method 4: User specified parameters through the GUI tabs}

The last method of entering data is to manually enter information on each of the input tabs of the GUI. Alternatively, the user can load data in the various tabs using the methods described earlier, and then manually edit the data as appropriate for their needs. All information must be present in all required locations. If a required entry is left blank, a warning message, similar to the one shown in Figure 3.1, will be displayed when the simulation is executed.

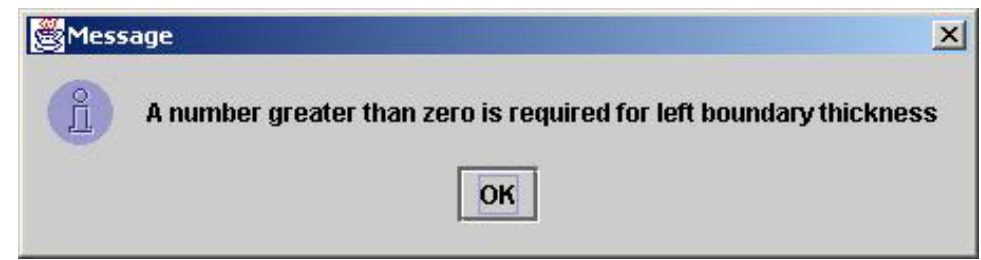

Figure 3.1: INPUT Error Dialog Box

\subsection{Performing a simulation}

Once the required information is specified on the input tabs, a simulation is performed when the Execute option under the "Run" pull-down menu is selected. This action 
begins a check of the supplied data for completeness and consistency, creates the INPUT file, and initiates the solver. The user can then follow the progress of the simulation in the DOS shell status window, show in Figure 3.2.

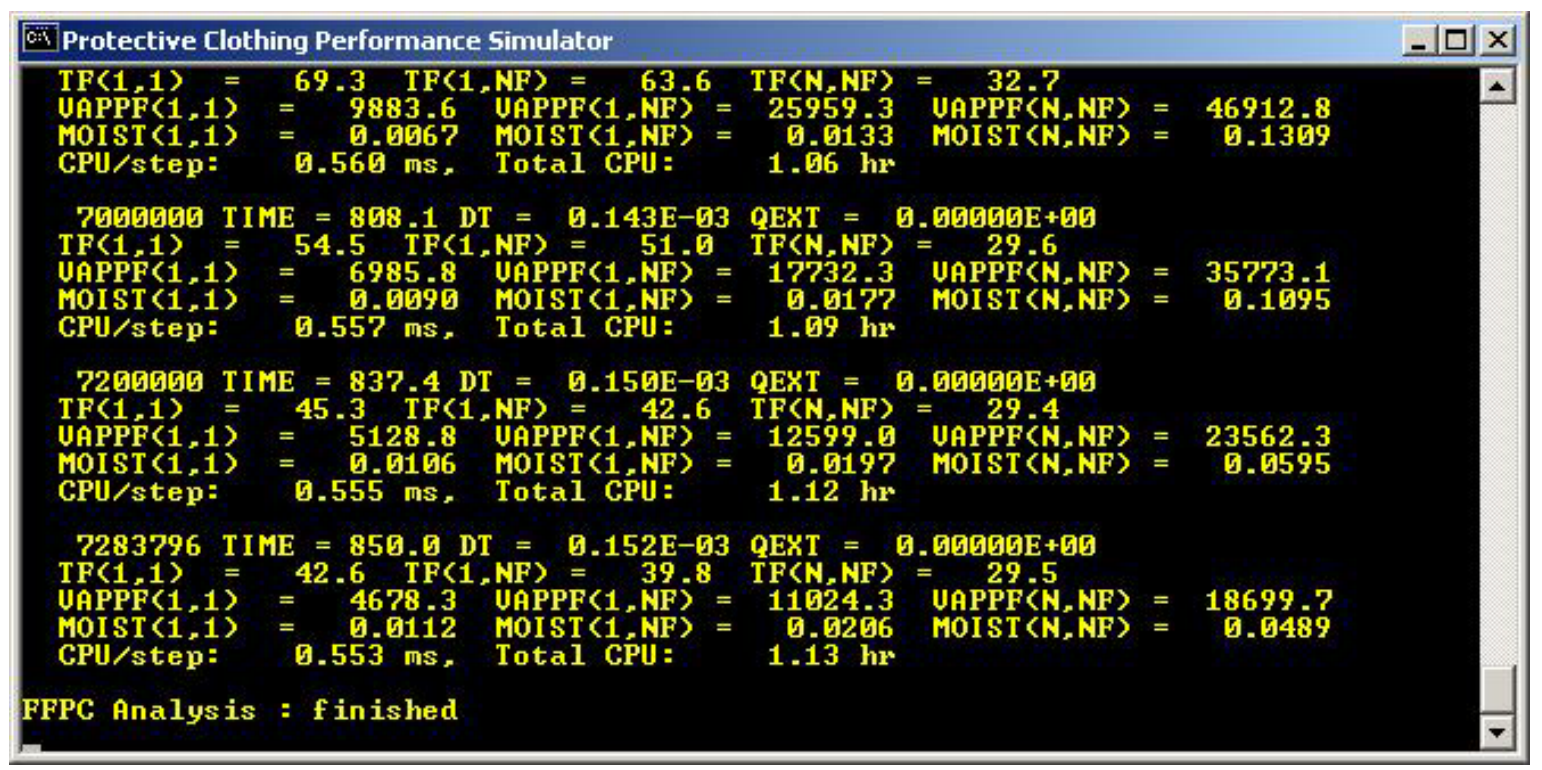

Figure 3.2: The DOS shell status window of a simulation

Information is printed to this window at fixed points of the solution by the Fortran solver. Information regarding the elapsed time; temperature, vapor pressure and moisture function values at three points along the fabric; and the CPU load for the simulation are displayed. This window will also notify the user when the simulation is completed, by displaying the message "FFPC Analysis : finished" when the solver stops.

While the solution is being computed, the user can conduct any post-processing of the data. This post-processing uses data that is recorded in a series of ASCII text files, created when the simulation is initiated. Graphs are created from the data contained in these files. The format of the output data and a description of the graphs available from PCPS are contained in Chapter 4Chapter 4:. 


\section{Chapter 4: How to analyze the results of a simulation}

The raw data results from a PCPS simulation are stored in a series of ASCII formatted output files. These files have the extension.DATA and are stored in the directory from where PCPS is being run. The data stored within these files can be opened from the GUI by selecting Print \& Save Output from the File drop-down menu. This opens a separate display from which the file can be viewed, printed or saved to a different name / location. The graphs available from within the GUI also use the data stored in these files.

\subsection{Using the GUI to analyze the results}

Contained within the GUI is a series of graphs used to view and analyze the results of a simulation. The graphs are visualized by going to the "Graph" pull down menu and selecting any one of the nine graphs. The first six graphs are static plots of important physical quantities versus time. The last three graphs are animated plots showing both the spatial dependence and temporal evolution. The graphs may be visualized at any time following the start of a simulation. When a graph is visualized, the GUI will read all the data stored in the relevant data file and plot it in a new window. While a simulation is still in the process of executing, data will continue to be written to all of the output files until the execution ends. Static graphs do not update automatically (as new data is generated during a simulation), but can be closed and then reopened to update the results. These graphs can be printed directly from the display window, or saved in a graphical format (.jpg) for later use. Animated graphs update dynamically as the simulation proceeds. Individual frames can be printed or saved in a graphical format (.jpg). We next give a brief description of the various graphs and animations that can be visualized using the "Graph" pull down menu. 
4.1.1 Temperature (front $\&$ back faces) versus Time

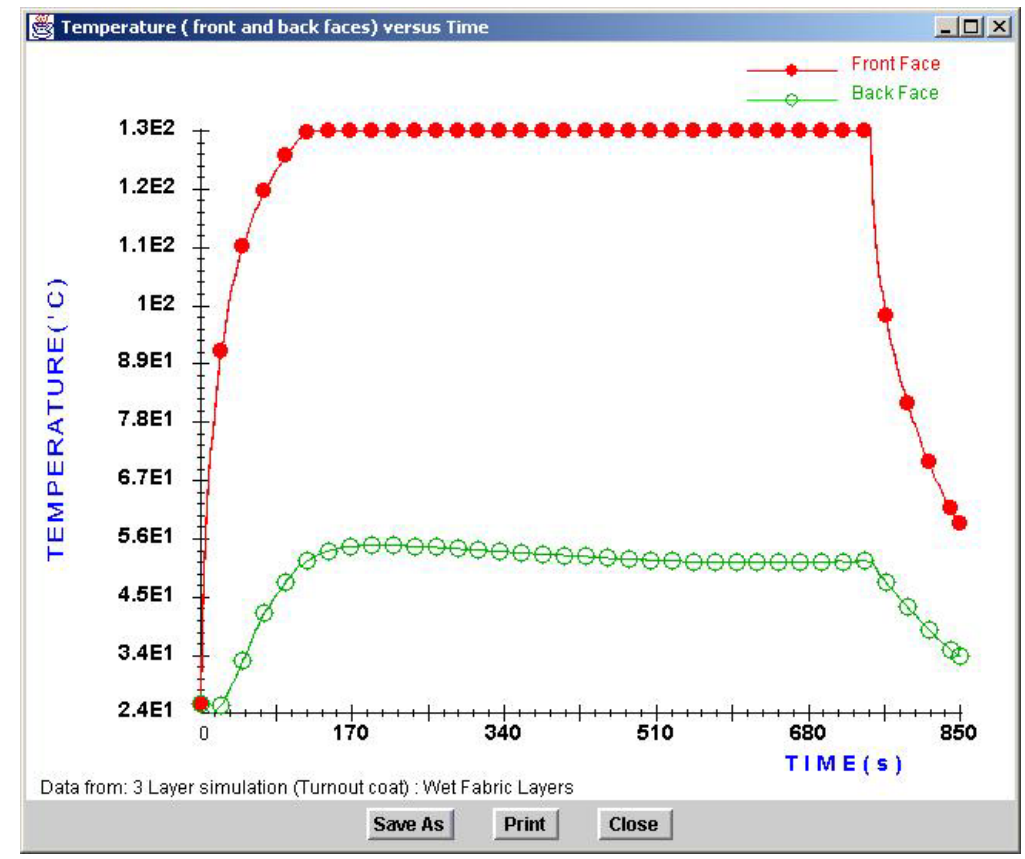

Figure 4.1: Sample "Temperature (front \& back faces) versus Time" graph

The Temperature (front $\&$ back faces) versus Time graph (Figure 4.1) displays the temperature (in degrees Celsius) of the far left and the far right faces of the multi-layered fabric. The front (left) face is the face exposed to the radiative flux from the fire. The back (right) face abuts the item or space being protected from the fire.

\subsubsection{Temperature (all faces) versus Time}

The Temperature (all faces) versus Time graph (Figure 4.2) displays not only the temperature at the front and back faces of the multi-layered material, but the temperature at all interior faces of the material as well. The total weight of water contained within the fabric layers is also plotted. As previously discussed, this value is calculated for a standardized 10 inch by 11 inch rectangular piece of material. 


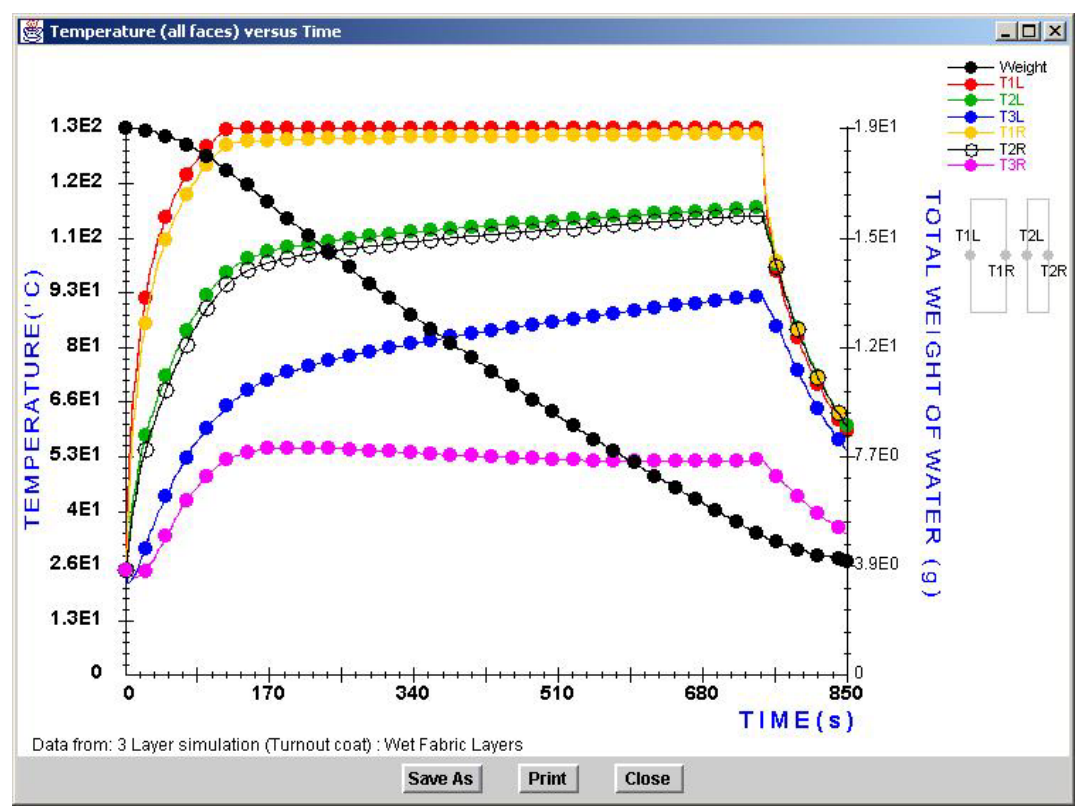

Figure 4.2: Sample "Temperature (all faces) versus Time" graph

\subsubsection{Burn Injury}

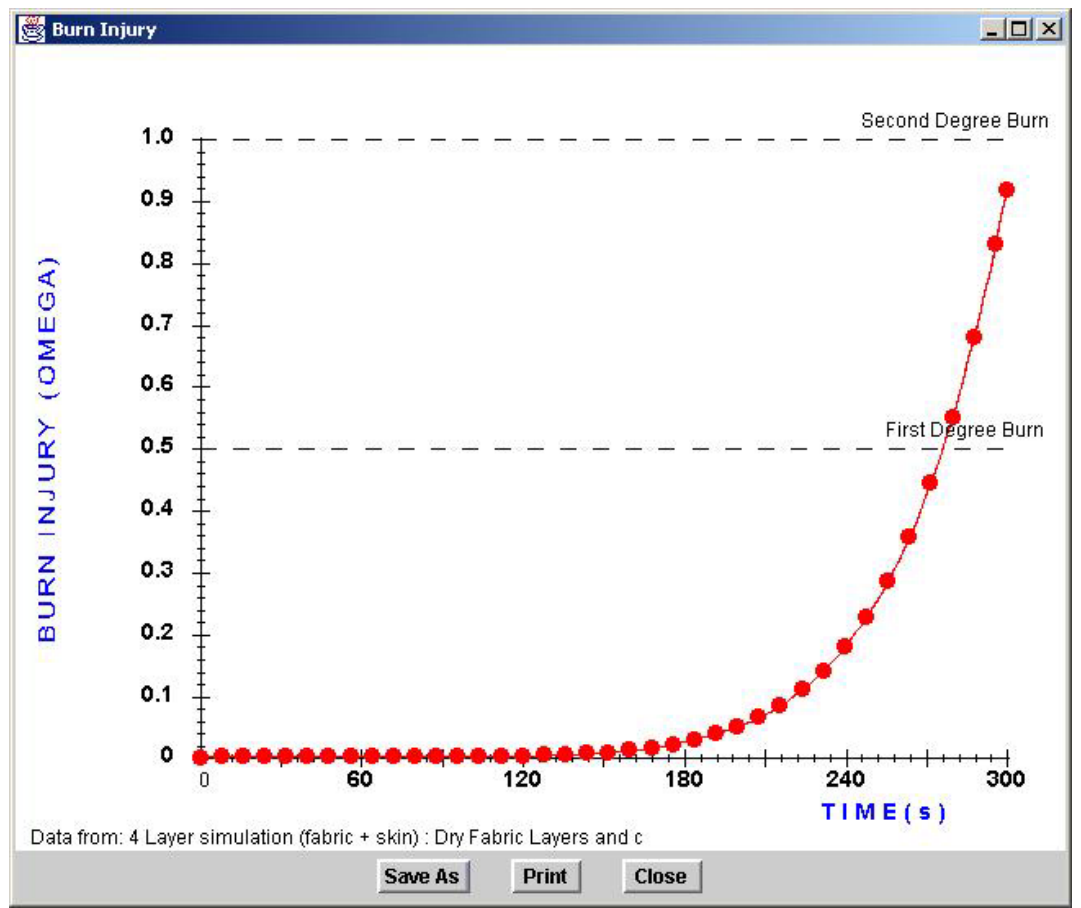

Figure 4.3: Sample "Burn Injury" graph

The Burn Injury graph (Figure 4.3) plots the cumulative damage function over the time of the simulation. First and second-degree burns are defined as $\Omega=0.5$ and $\Omega=1.0$ respectively. These limits are included as dashed lines on the graph. The value of $\Omega$ is only calculated when skin modeling is included in the simulation. 


\subsubsection{Convective Flux versus Time}

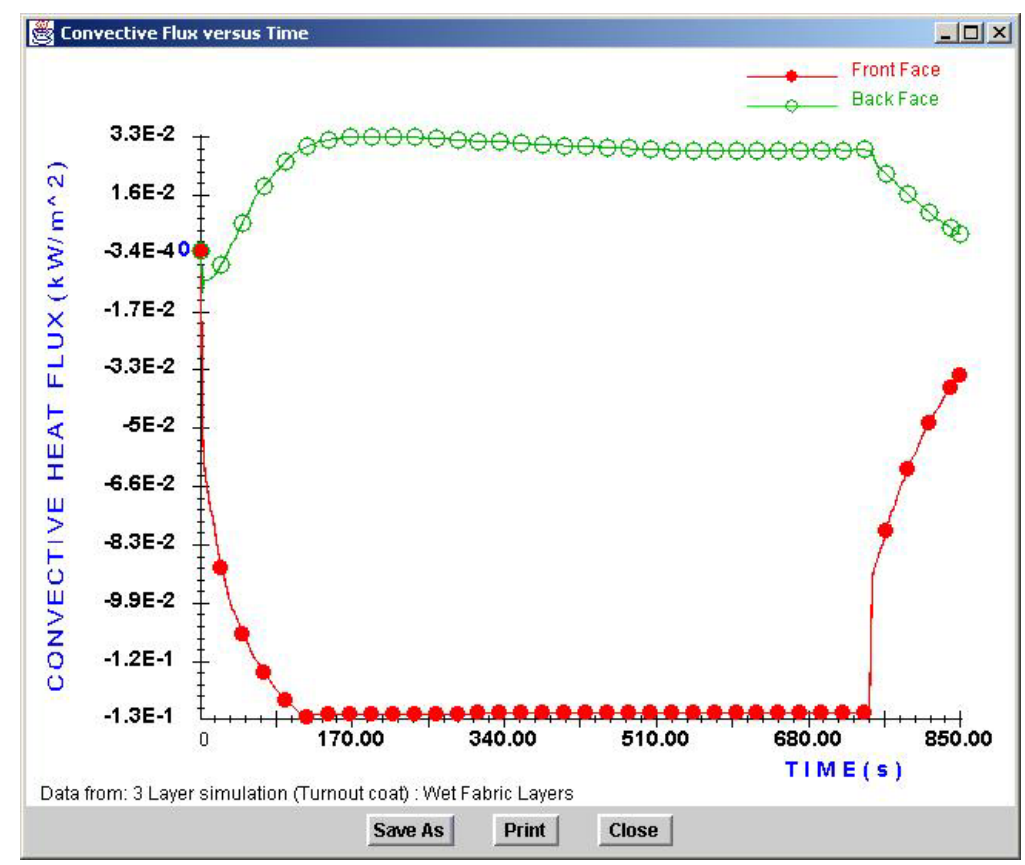

Figure 4.4: Sample "Convective Flux versus Time" graph

The Convective Flux versus Time graph (Figure 4.4) displays the convective (or conductive depending upon the case) flux of heat energy at the front and back faces of the multi-layered fabric as a function of time. Flux is positive for heat flow from left to right of the composite material.

\subsubsection{Radiative Flux versus Time}

The Radiative Flux versus Time graph (Figure 4.5) displays the radiative flux of heat energy at the front and back faces of the multi-layered fabric as a function of time. Flux is positive from for radiation from left to right of the composite material. 


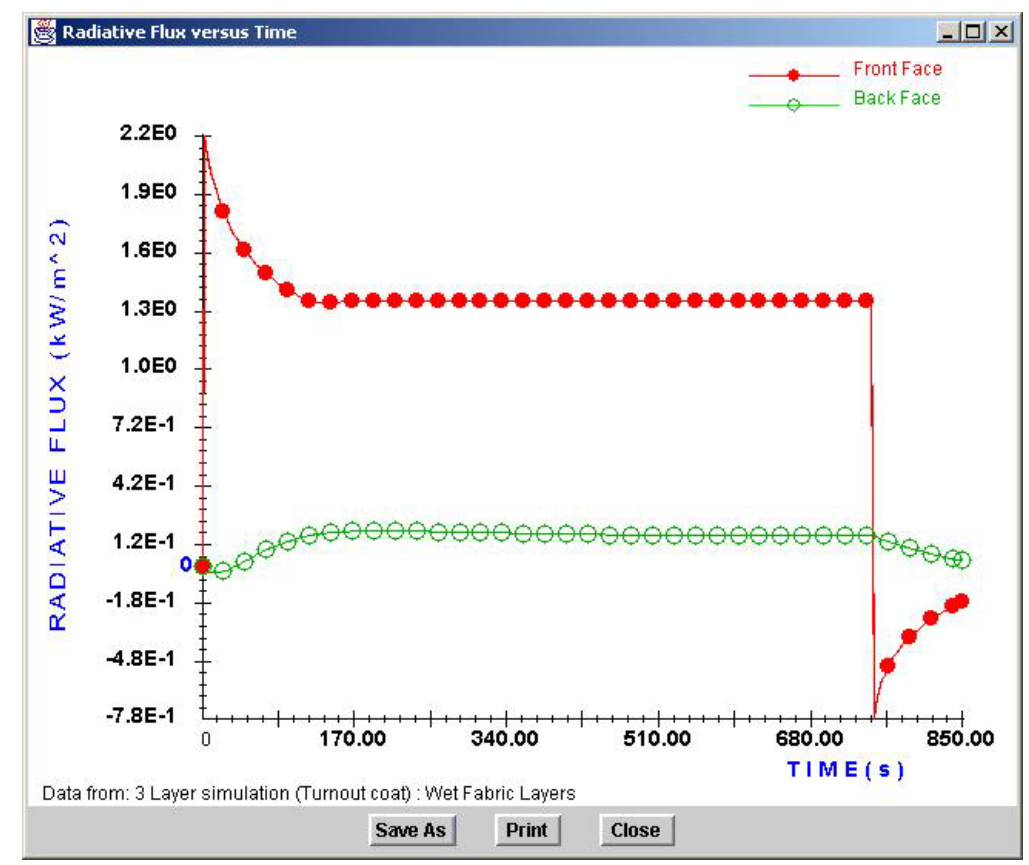

Figure 4.5: Sample "Radiative Flux versus Time" graph

\subsubsection{Mass Flux versus Time}

The Mass Flux versus Time graph (Figure 4.6) displays the mass flux of water vapor at the front and back faces of the multi-layered fabric as a function of time. Flux is positive for net mass flux from left to right of the composite material.

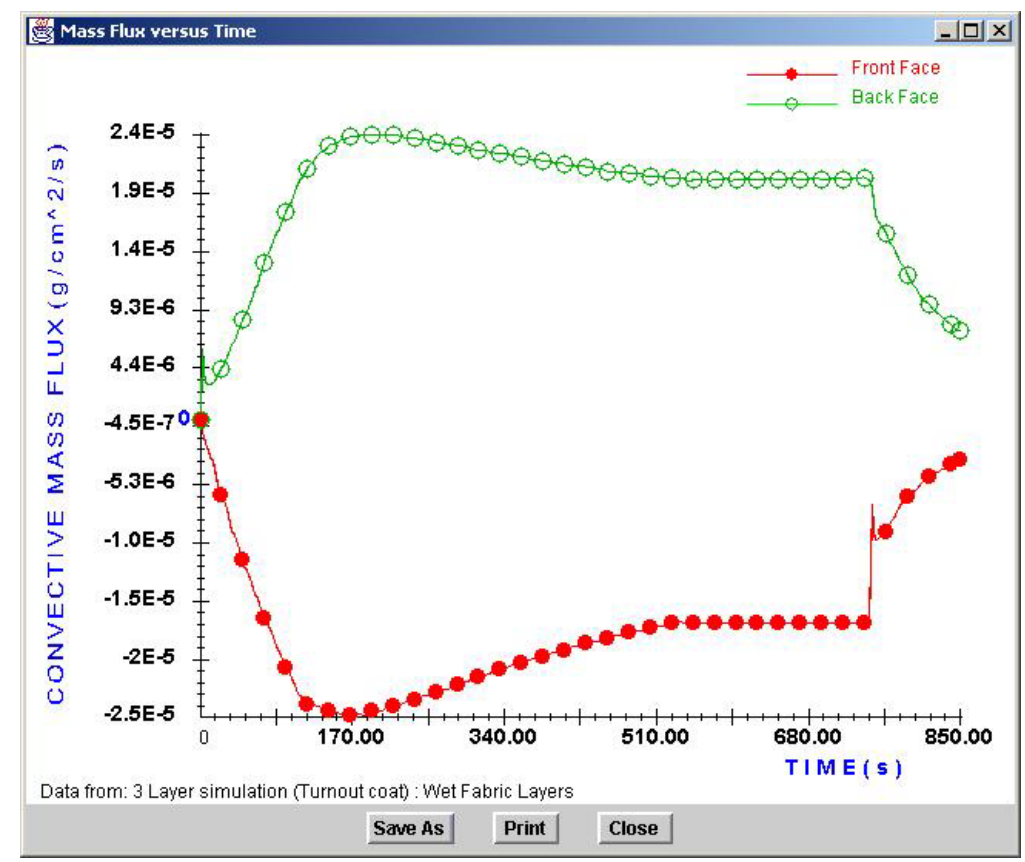

Figure 4.6: Sample "Mass Flux versus Time" graph 


\subsubsection{Animation of Flow Variables}

The Animation of Flow Variables graph (Figure 4.7) displays four flow variables for the entire width of the multi-layered fabric. The displayed variables are Temperature, Vapor Pressure, Moisture Content and Density. The variables are displayed as animations. Through the buttons at the bottom the user can start, stop or speed up the animation. The individual frames can be saved or printed by pressing the appropriate button at the bottom of the plot.

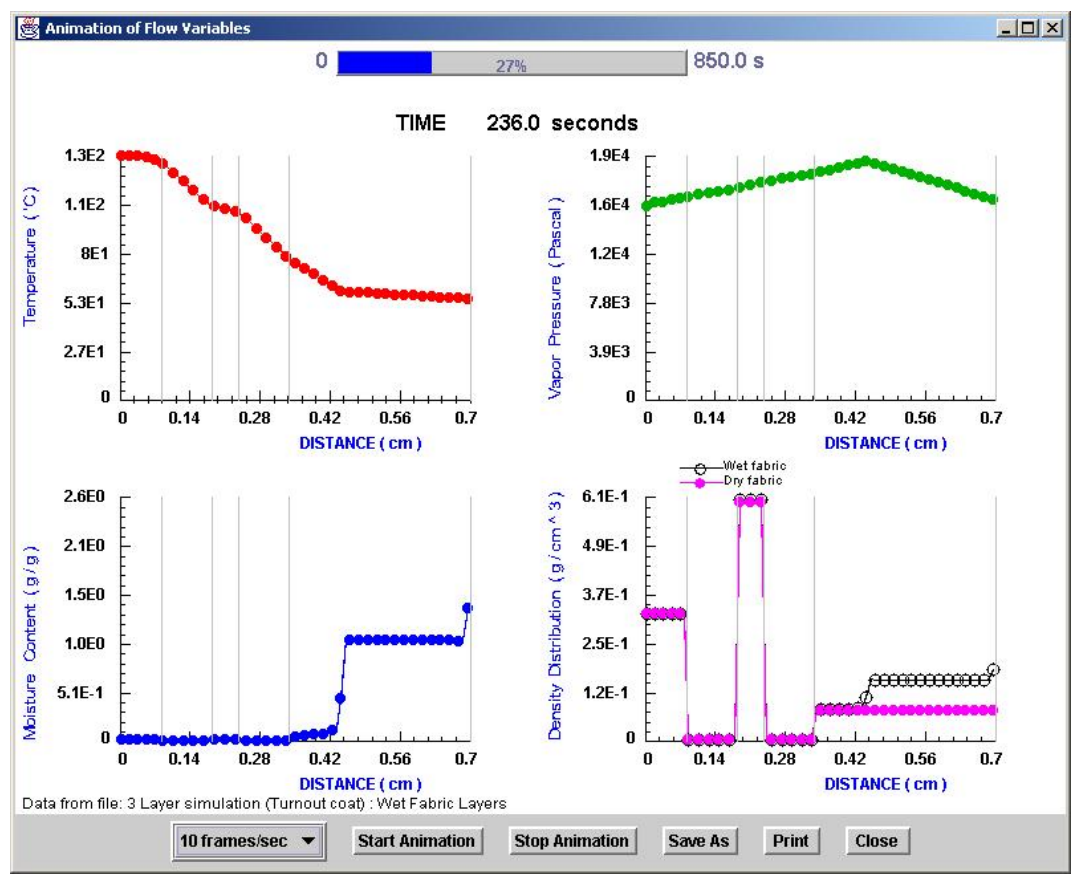

Figure 4.7: Sample "Animation of Flow Variables" graph

\subsubsection{Animation of Terms in Energy Equation}

The Animation of Terms in the Energy Equation graph (Figure 4.8) displays the individual contributions to the conservation of energy for the entire width of the multilayered fabric. The five terms presented are conduction, radiation, water vapor diffusion, moisture accumulation, and metabolism. The metabolism term is used when modeling skin and represents the contribution of the body to the transfer of heat. The variables are displayed as animations. Through the buttons at the bottom the user can start, stop or speed up the animation. The entire animated sequence cannot be saved as a single file, however, individual frames can be saved or printed by pressing the appropriate button. 


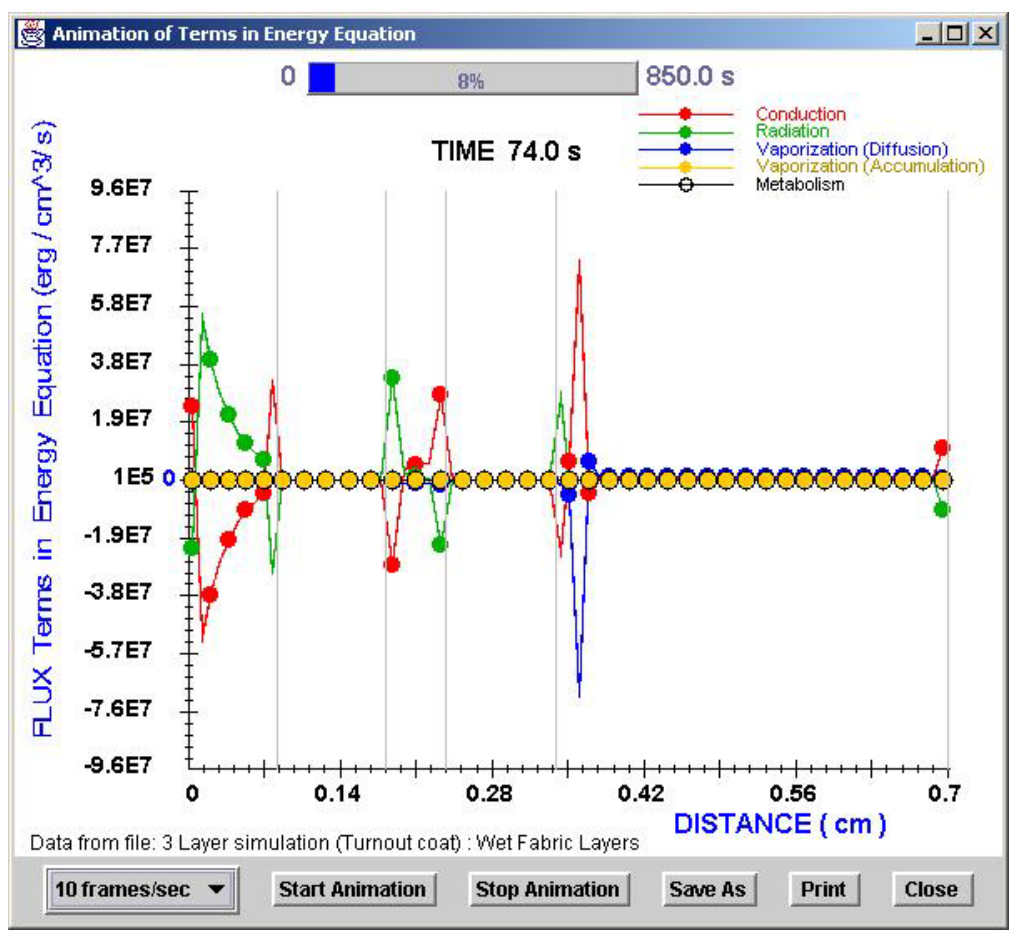

Figure 4.8: Sample "Animation of Terms in Energy Equation" graph

\subsubsection{Animation of Terms in Mass Equation}

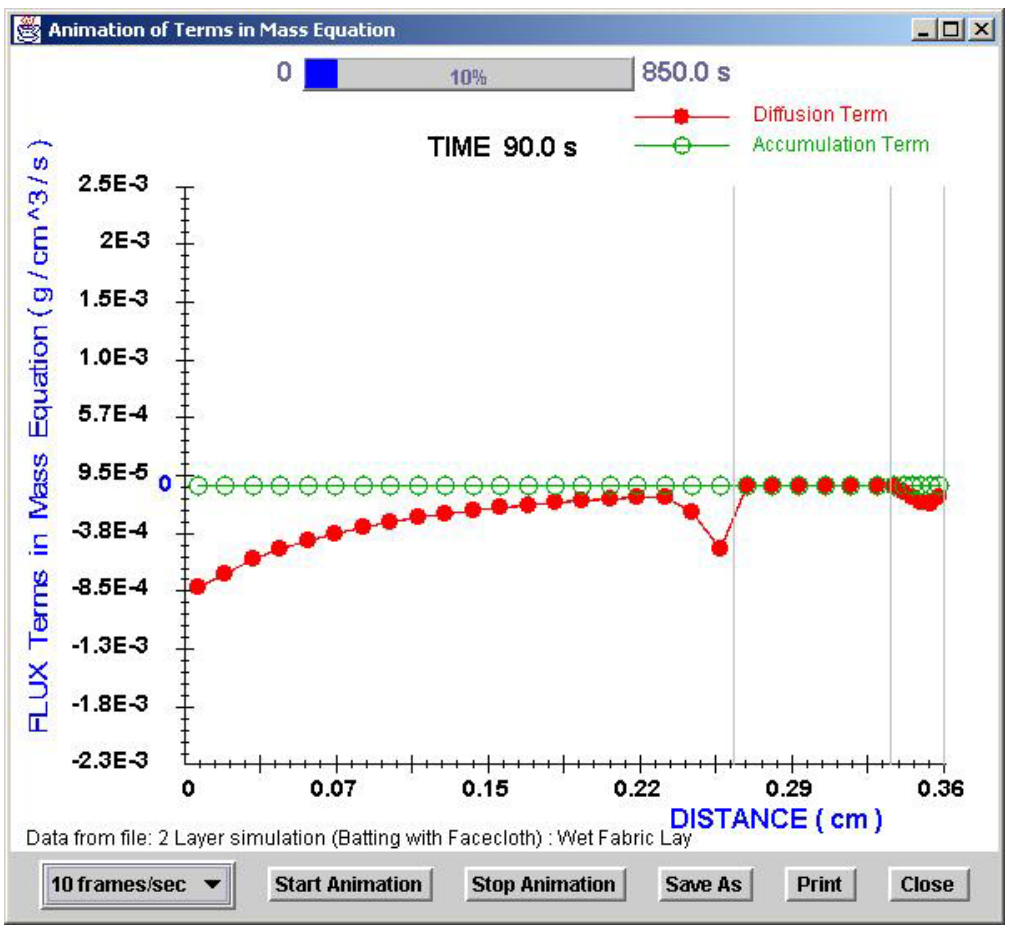

Figure 4.9: Sample "Animation of Terms in Mass Equation" graph 
The Animation of Terms in the Mass Equation graph (Figure 4.9) displays the individual contribution to the conservation of mass for the entire width of the multilayered fabric. The two terms presented are water vapor diffusion and moisture accumulation. The variables are displayed as animations for the length of the simulation. Through the buttons at the bottom the user can start, stop or speed up the animation. The entire animated sequence cannot be saved as a single file, however individual frames can be saved or printed by pressing the appropriate button.

\subsection{Using the Output DATA files to analyze the results}

The raw data results from a PCPS simulation are stored in a series of ASCII formatted output files. The data stored within these files can be opened in any text editor or from the GUI by selecting Print \& Save Output from the "File" drop down menu. These files can also be edited in any text editor or imported into various graphics packages for additional post-processing (if desired). We next describe the format and contents of the various output data files that are created by the PCPS software.

\subsubsection{TEMP.DATA}

The TEMP.DATA file stores the temperature at the faces of each layer of fabric, the total weight of water contained within the fabric layers, and the cumulative damage to skin. The data is organized in rows, with each row corresponding to a given point in time. Each row is comprised of formatted columns of data,

\section{TIME T_LEFT_01 ... T_RIGHT_01 ... WEIGHT OMEGA}

where,

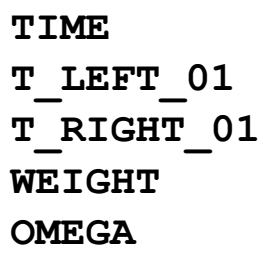

Output time

Temperature of left faces of fabric layers

Temperature of right faces of fabric layers

Cumulative weight of water in the fabric layers

Cumulative skin damage value

The cumulative weight of water in the fabric layers is calculated by assuming a rectangular piece of fabric of dimension $25.4 \mathrm{~cm} \times 27.9 \mathrm{~cm}$ (10 inches $\times 11$ inches). The cumulative skin damage value is used to predict superficial first and second-degree burns.

The first few lines contain administrative information for the file. The title of the simulation is displayed, followed by descriptions of the columns stored data. The data is in fixed width format, and can be imported into almost any graphing package. 
Time units for this file are seconds, temperature units are degrees Celsius, and weight units are grams.

\subsubsection{COND_FLUX.DATA}

The COND_FLUX.DATA file stores information concerning the convective or conductive flux of energy at the faces of the multi-layered material. The choice of convective or conductive flux is made in the declarations of the simulation. The data is organized in rows, with each row corresponding to a given point in time. Each row is comprised of data in column format,

TIME QCONDB_L_01 $\ldots$. QCONDB_R_01 $\ldots$. HCF $\quad$ HCB

where,

$\begin{array}{ll}\text { TIME } & \text { Output time } \\ \text { QCONDB_L_01 } & \text { Conductive/Convective flux into the left face of layer 1 } \\ \text { QCONDB_R_01 } & \text { Conductive/Convective flux out of the right face of layer 1 } \\ \text { HCF } & \text { Heat transfer parameter at left boundary } \\ \text { HCB } & \text { Heat transfer parameter at right boundary }\end{array}$

The continuation ellipses denote the presence of additional layers (if present), expanding the length of the line. Flux is considered positive in the direction from left to right.

The first few lines contain administrative information for the file. The title of the simulation is displayed, followed by descriptions of the columns stored data. The data is in fixed width format, and can be imported into most graphing packages.

Time units for this file are seconds, heat flux units are erg $/ \mathrm{s}-\mathrm{cm}^{2}$ and the units of the heat transfer parameter are erg $/ \mathrm{s}-{ }^{0} \mathrm{C}-\mathrm{cm}^{2}$.

\subsubsection{RAD_FLUX.DATA}

The RAD_FLUX.DATA file stores information concerning the radiative flux of energy at the faces of the multi-layered material from both the external fire source and the interlayer radiation arising from temperature differences between the fabric layers. The data is organized in rows, with each row corresponding to a given point in time. Each row is comprised of data in column format,

TIME QRADB_L_01 $\ldots .$. QRADB_R_01 $\ldots .$. QR12_L_01 $\ldots$. QR12_R_01 $\ldots$. QLS QRS

where,

TIME

QRADB_L_01 QRADB R 01 QR12_ I $-\overline{0} 1$
Output time (s)

External radiative flux into the left face of layer 1

External radiative flux out of the right face of layer 1

Inter-layer radiative flux into the left face of layer 1 


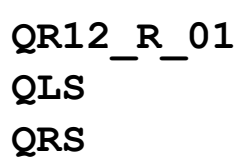

Inter-layer radiative flux out of the right face of layer 1

Net radiative flux into the left boundary

Net radiative flux out of the right boundary

The continuation ellipses denote the presence of additional layers (if present), expanding the length of the line. Fabrics in PCPS have been modeled to allow for absorption, reflection and transmission of incident radiation, thus the need for radiative fluxes on all faces. Flux is considered positive in the direction from left to right.

The first few lines contain administrative information for the file. The title of the simulation is displayed, followed by descriptions of the columns stored data. The data is in fixed width format, and can be imported into most graphing packages.

\subsubsection{MASS_FLUX.DATA}

The MASS_FLUX.DATA file stores information concerning the flux of water vapor at the faces of the multi-layered material. The data is organized in rows, with each row corresponding to a given point in time. Each row is comprised of data in column format,

\section{TIME MTRANB_L_01 $\ldots$ MTRANB_R_01 $\ldots$}

where,

$\begin{array}{ll}\text { TIME } & \text { Output time } \\ \text { MTRANB_L_01 } & \text { Mass flux at the left face of layer 1 } \\ \text { MTRANB_R_01 } & \text { Mass flux at the right face of layer 1 }\end{array}$

The continuation ellipses denote the possible presence of additional layers, expanding the line. Flux is considered positive in the direction from left to right.

The first few lines contain administrative information for the file. The title of the simulation is displayed, followed by descriptions of the columns stored data. The data is in fixed width format, and can be imported into most graphing packages.

Time units for this file are seconds and mass flux units are $\mathrm{gm} / \mathrm{s}-\mathrm{cm}^{2}$.

\subsubsection{VAR.DATA}

The file VAR.DATA stores the data used to produce the graph, "Animation of Flow Variables." It therefore contains information for all nodes as a function of time. The general format lists the result variables as a set for a given instant in time. The sets are stored in column format with the first column being the spatial coordinate and subsequent columns the results data,

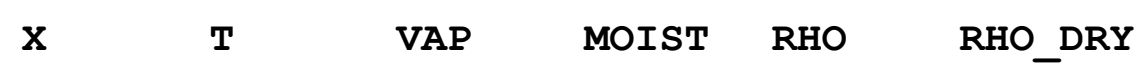

where, 


$\begin{array}{ll}\mathbf{X} & \text { Spatial coordinate } \\ \mathbf{T} & \text { Temperature } \\ \text { VAP } & \text { Vapor pressure } \\ \text { MOIST } & \text { Moisture distribution function } \\ \text { RHO } & \text { Wet density } \\ \text { RHO_DRY } & \text { Dry density (does not vary with time) }\end{array}$

The sets are arranged from time $\mathrm{t}=2.0$ seconds to the final simulation time in increments of 2 seconds. Because this file contains multiple variables over two dimensions (space and time), the post-processing is typically more involved to extract data from this file.

Spatial units for this file are centimeters, temperature units are degrees Celsius, vapor pressure units are Pascal, moisture distribution units are gram moisture / gram fabric, and density units are gram / centimeter ${ }^{3}$.

\subsubsection{QDIV.DATA}

The file QDIV.DATA stores the data used to produce the graph, "Animation of Terms in Energy Equation." The format is of the same form as that of the file VAR.DATA. Data is arranged in sets with each set corresponding to a given instant in time. This file stores the energy flux as a function of time and space. The flux is decomposed into conduction, radiation, water vapor diffusion, moisture accumulation and skin (body) metabolism.

The columns of each time set are,

\section{$\mathrm{X}$ DIVQC DIVQR DIVMCF DIVPDT GM}

where,

$\begin{array}{ll}\mathbf{X} & \text { Spatial coordinate } \\ \text { DIVQC } & \text { Conductive flux } \\ \text { DIVQR } & \text { Radiative flux } \\ \text { DIVMCF } & \text { Flux of energy due to water vapor diffusion } \\ \text { DIVPDT } & \text { Flux of energy due to moisture accumulation } \\ \text { GM } & \text { Flux of energy due to metabolism effects }\end{array}$

The sets are arranged from time $t=2.0$ seconds to the final simulation time in increments of 2 seconds. Because this file contains multiple variables over two dimensions (space and time), the post-processing is typically more involved to extract data from this file.

Spatial units for this file are centimeters, and units of flux are erg / second-centimeter ${ }^{3}$.

\subsubsection{MDIV.DATA}

The file MDIV.DATA stores the data used to produce the graph, "Animation of Terms in Mass Equation." The format is of the same form as that of the file VAR.DATA. Data is arranged in sets with each set corresponding to a given instant in time. This file stores 
the mass flux as a function of time and space decomposed into water vapor diffusion and moisture accumulation.

The columns of each time set are,

\section{$\mathrm{X} \quad$ DIVMCF DIVPDT}

where,
X Spatial coordinate
DIVMCF Mass flux due to vapor diffusion
DIVPDT Mass flux due to accumulation

The sets are arranged from time $t=2.0$ seconds to the final simulation time in increments of 2 seconds. Because this file contains multiple variables over two dimensions (space and time), the post-processing is typically more involved to extract data from this file.

Spatial units for this file are centimeters, and units of flux are gram / second-centimeter ${ }^{3}$. 


\section{Chapter 5: A sample case study using PCPS}

Presented here is a sample case study of two possible scenarios. The study is presented to walk the user through the type of analysis that can be performed using PCPS. Additionally, the capability of PCPS is demonstrated and the importance of moisture (water) transport in the analysis of fabrics subject to radiative heat flux is shown.

In both cases, a single thermal barrier will be used to provide skin protection from a fire. A $1 \mathrm{~mm}(0.039 \mathrm{inch})$ air gap is present between the thermal barrier and the three layers of skin. The fabric begins initially at $25.22{ }^{0} \mathrm{C}\left(77^{0} \mathrm{~F}\right)$ and is subjected to a radiative flux of $2.5 \mathrm{~kW} / \mathrm{m}^{2}$. Convection from the left boundary is to an ambient at $29.3{ }^{0} \mathrm{C}\left(85^{0} \mathrm{~F}\right)$ and a vapor pressure of 200 Pascal (5\% relative humidity). Both situations considered will run for 300 seconds, with no post-radiative cooling.

\subsection{Dry Fabric}

The first scenario is that of a dry fabric. A small moisture distribution of 0.1 grams (water) / grams (fabric) is present throughout the entire fabric. To begin the analysis, the user should first go to the Fabric / Skin Layer tab and provide the required information concerning the individual layers considered. The user should first enter a title for the simulation, which here is called "4 layer simulation (fabric + skin) : Dry Fabric layers and Low Flux." The title used here provides sufficient information to fully describe the situation being considered. Directly underneath the title the user should then enter the total number of fabric / skin layers under consideration. As the title states this should be set to four (fabric, epidermis, dermis and subcutaneous tissue).

Once the total number of layers has been entered, an equal number of lines becomes active below this entry. Each line corresponds to a layer of fabric / skin and must be properly defined. Beginning with the outermost layer, the down arrow (upside down triangle), displays a pull-down menu of the available fabric / skin materials. For this scenario, the user should select "Aralite." Once the material for the fabric has been chosen, then next step is to provide the resolution desired through the fabric. As the outermost layer is subjected to the greatest heat flux from the radiative source it is good 
practice to utilize greater resolution for this layer. For this scenario a value of 30 nodes will provide adequate resolution. Finally, the initial temperature of the fabric is set to a room temperature ambient of $25.22{ }^{\circ} \mathrm{C}$.

In the same way as for the outermost thermal barrier layer, the remaining layers should also be filled in. These layers correspond to the three layers of skin which are being modeled and must be entered in the correct order. Beginning with the Epidermis, a resolution of 10 nodes is entered, as is the initial body temperature of $37.0{ }^{\circ} \mathrm{C}$. The next layer of the skin is the Dermis with a resolution of 20 nodes and again the initial body temperature of $37.0{ }^{\circ} \mathrm{C}$. The last layer of skin is the Subcutaneous Tissue with 20 nodes and an initial temperature of $37.0{ }^{\circ} \mathrm{C}$. When all data has been entered the Fabric / Skin Layer tab should look as shown in Figure 5.1.

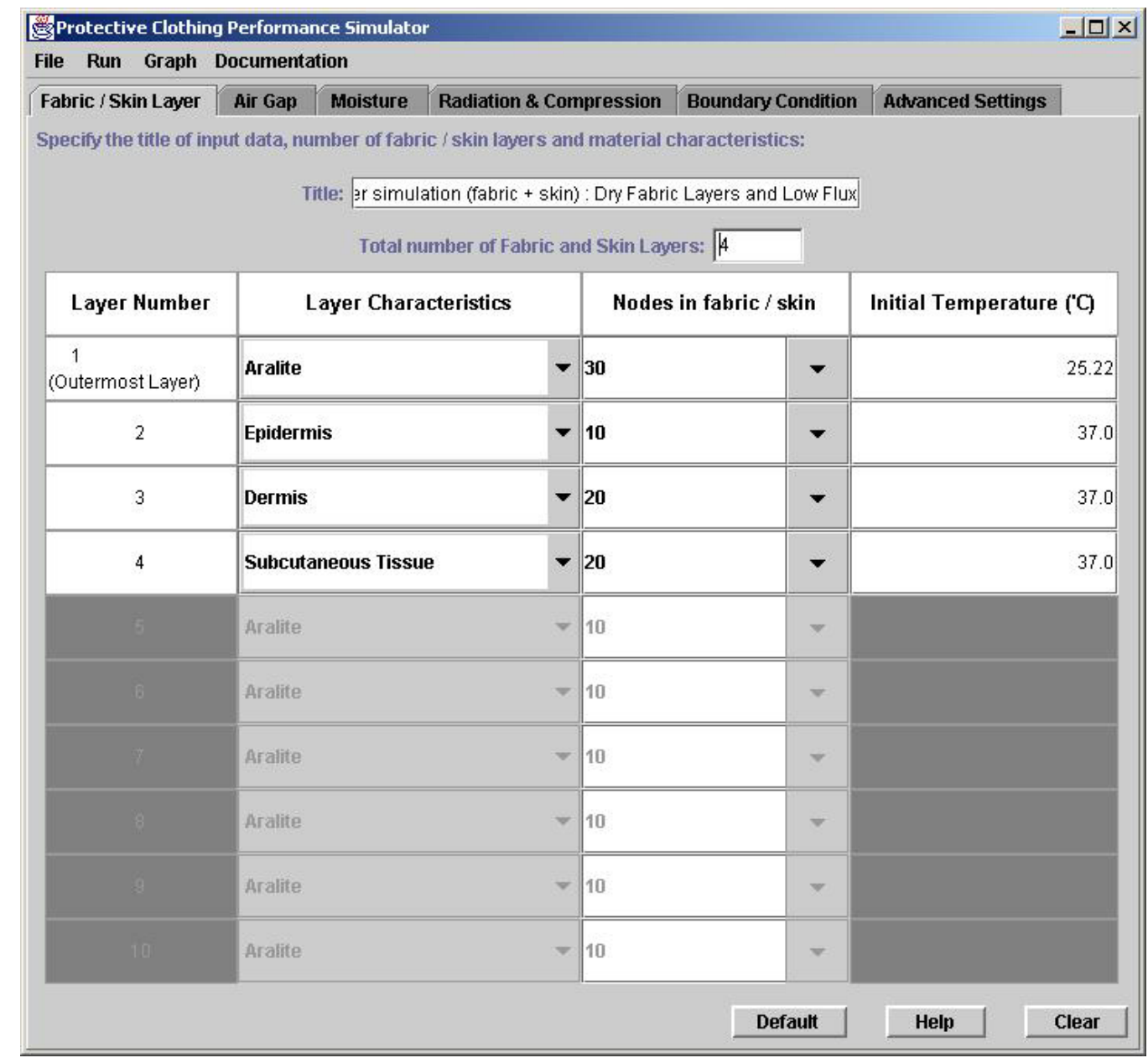

Figure 5.1: Fabric / Skin Layer tab for dry fabric case study

The user should now proceed to the next tab, the Air gap tab. Being a four layer simulation, three air gaps are active and able to be filled in. For this case, air gap number one is between fabric / skin layers one and two and subsequently is the gap between the 
thermal barrier and the top layer of skin. For this case a modest gap of $1 \mathrm{~mm}(0.1 \mathrm{~cm})$ is entered. Proceeding to the nodes, the user should manually enter a value of 5 nodes for this layer (note that this number does not exist under the pull-down but rather must be keyed in). This gap is initially at the room ambient temperature of $25.22{ }^{0} \mathrm{C}$. Being a dry fabric, the vapor pressure within this air gap is then set to a small value of $0.1 \mathrm{~Pa}$ (again note that this number does not exist under the pull-down but must be keyed in as well).

The second and third possible air gaps are between the skin layers. No air gap actually exists here and thus a value of 0 nodes should be entered in the Nodes in Air Gap section of the window. The remaining fields for these layers no longer are meaningful for a 0 node gap, however to pass the consistency check, these fields must have some numbers entered. The user can therefore enter any values desired here. When all fields have been properly filled in, the resulting tab should look as shown in Figure 5.2.

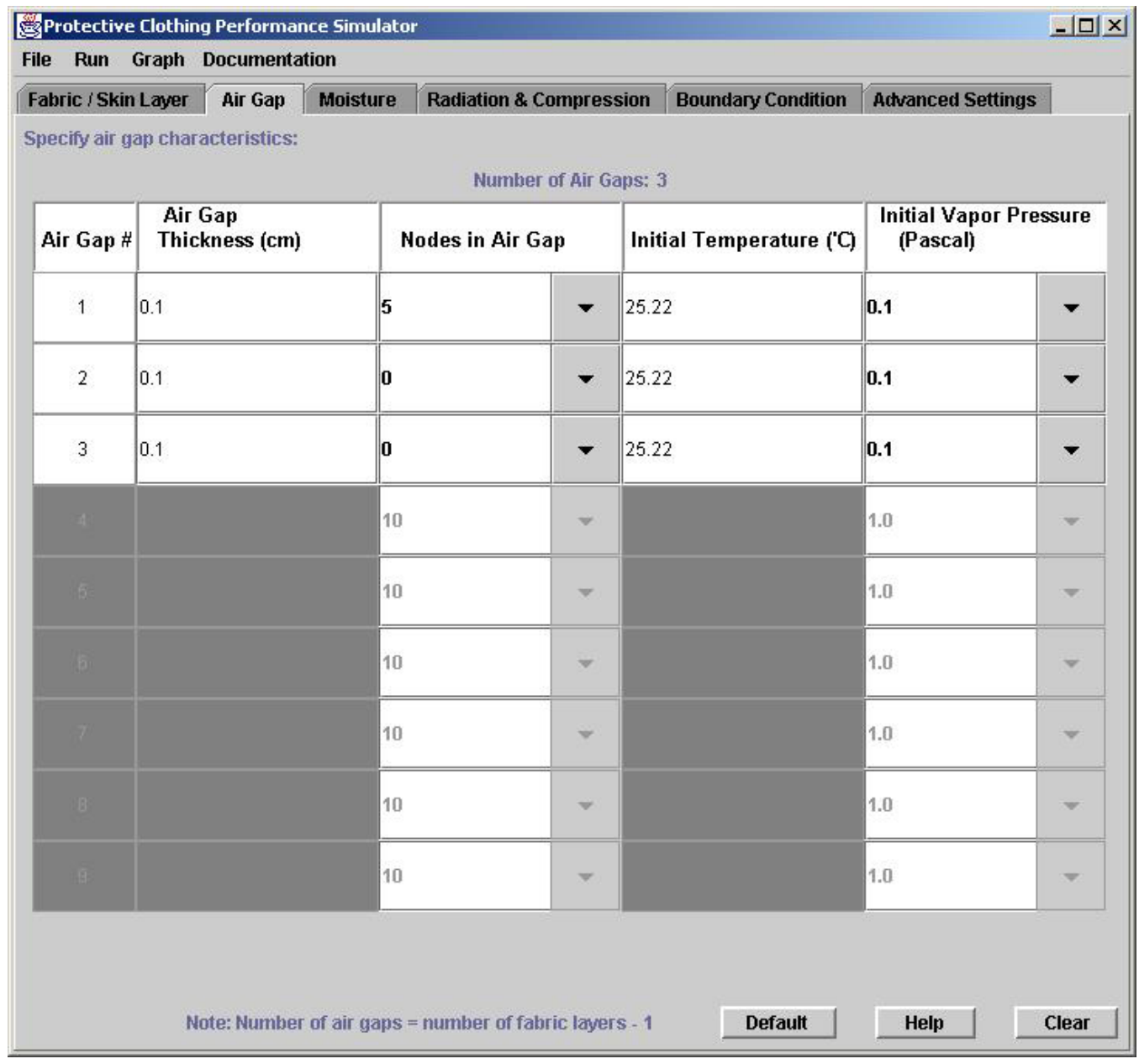

Figure 5.2: Air Gap tab for dry fabric case study

With the composition of the Fabric / Skin Layers and the Air Gaps, the user should now proceed to the third tab, the moisture tab. This tab provides initial moisture data for each of the fabric / skin layers. The mass transfer equation is not solved in each of the skin layers, and therefore no information is required for the skin layers, however the initial 
distribution of water is required for the thermal barrier. The scenario is for a dry fabric layer and therefore a small moisture distribution function of 0.01 grams (water) / grams (fabric) is considered constant through out the fabric. To enter this function the user should go to Layer \#1 and enter two points used to define the moisture distribution. Directly underneath this entry the two points should be entered. The first point is at $0 \%$ thickness and has a moisture distribution function of 0.01 . The second point entered is at $100 \%$ thickness and has a moisture distribution function of 0.01 . Once these values have been entered, a graph of the distribution is shown directly underneath.

Although no information is required for the Fortran solver, the consistency check requires a moisture distribution function to be defined for each layer. Any values can be entered, and therefore for fabric / skin layers two through four the same distribution function of 0.01 is entered uniformly across each layer as was done for the thermal barrier. Be sure to scroll to the right to fill in all required information for layer number four as well. When completed, the moisture tab should look as shown in Figure 5.3.

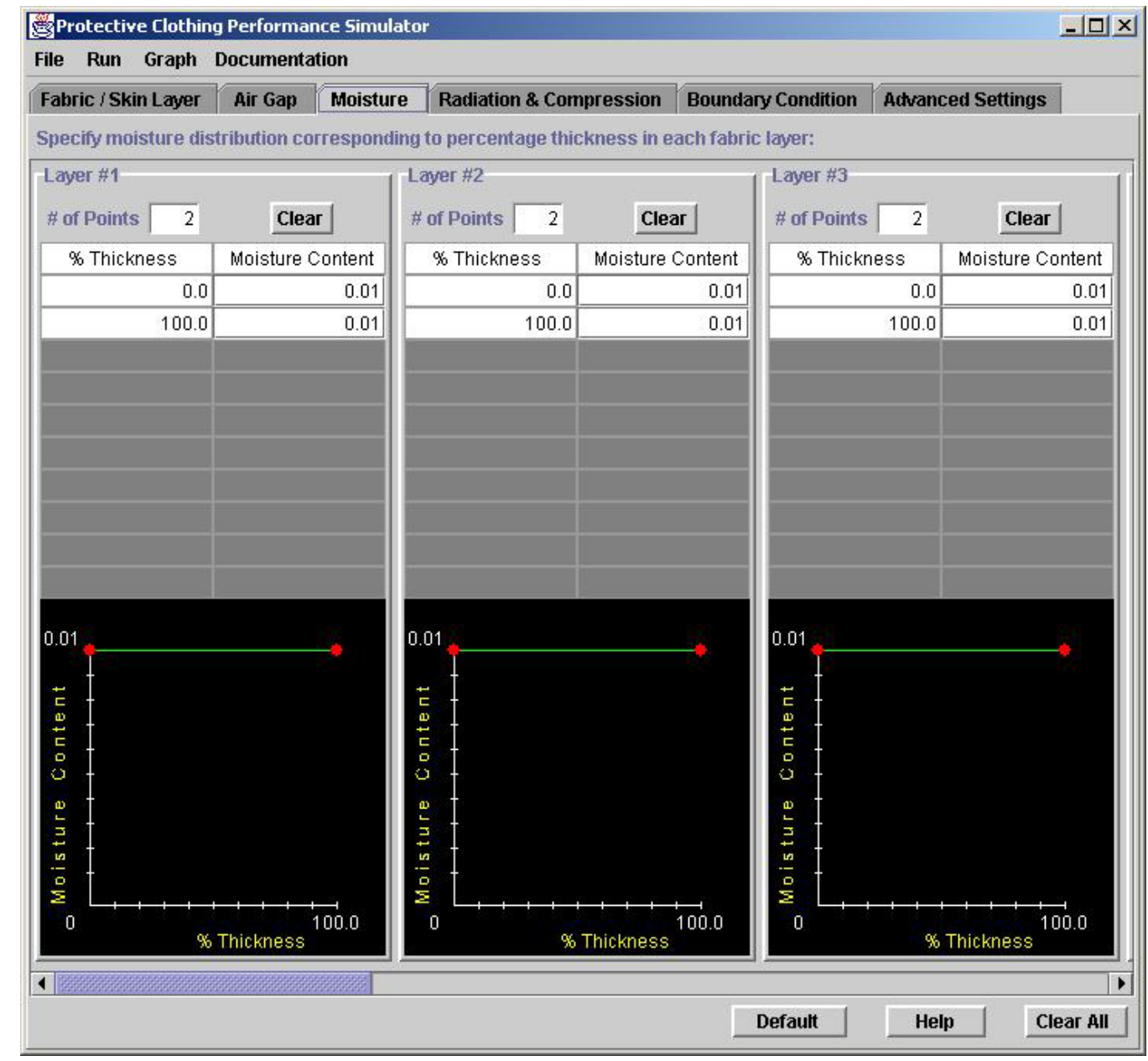

Figure 5.3: Moisture tab for dry fabric case study 
The next tab is the Radiation and Compression tab. Here the user should enter a value for the radiative flux incident upon the outermost fabric layer, taken here to be a relatively small value of $2.5 \mathrm{~kW} / \mathrm{m}^{2}$. The simulation time and the cutoff time must also be entered. For this case the simulation is run for $300 \mathrm{~s}$ and the cutoff time is also taken to be $300 \mathrm{~s}$. No compression of the fabric layers is considered. When completed, the tab should look as shown in Figure 5.4.

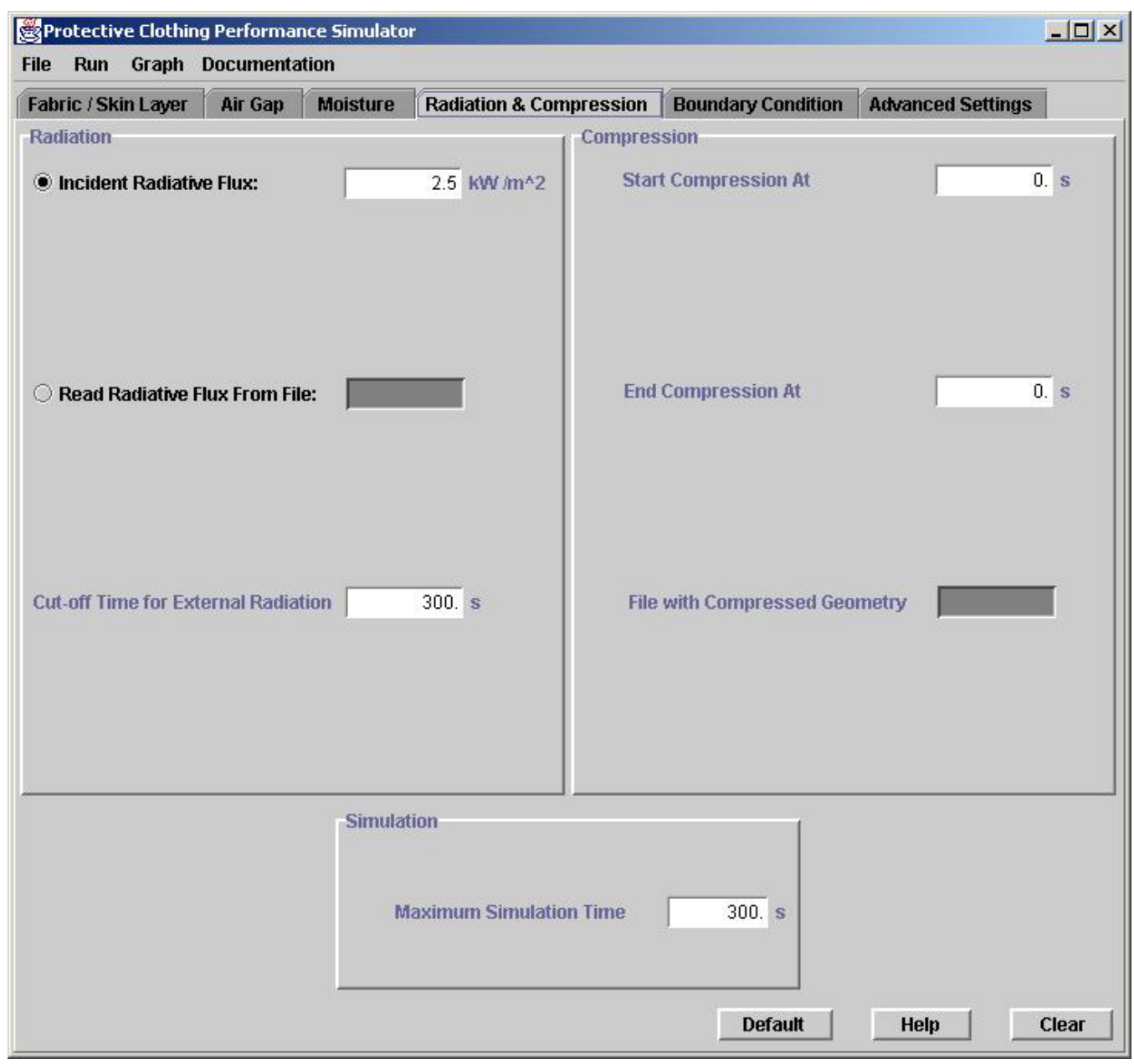

Figure 5.4: Radiation \& Compression tab for dry fabric case study

The next tab defines the boundary conditions for the simulation. For the left boundary, an ambient temperature of $29.33{ }^{\circ} \mathrm{C}$ is considered with vapor pressure of the ambient taken to be $200 \mathrm{~Pa}$ (corresponding to a $5 \%$ relative humidity for this temperature). In addition to the radiative flux, convective heat losses are also considered to the ambient by selecting "Yes" from the convective boundary pull down tab. Conductive losses are ignored in the simulation by selecting "No" from the conductive boundary pull down tab. Although this makes the boundary thickness and number of points considered in the boundary meaningless the consistency check requires values to be entered here. 
With the skin model included the entire series of right boundary conditions is also meaningless but must have some values entered. These values could be entered to be identical to the left boundary. When completed, the boundary condition tab looks as shown in Figure 5.5.

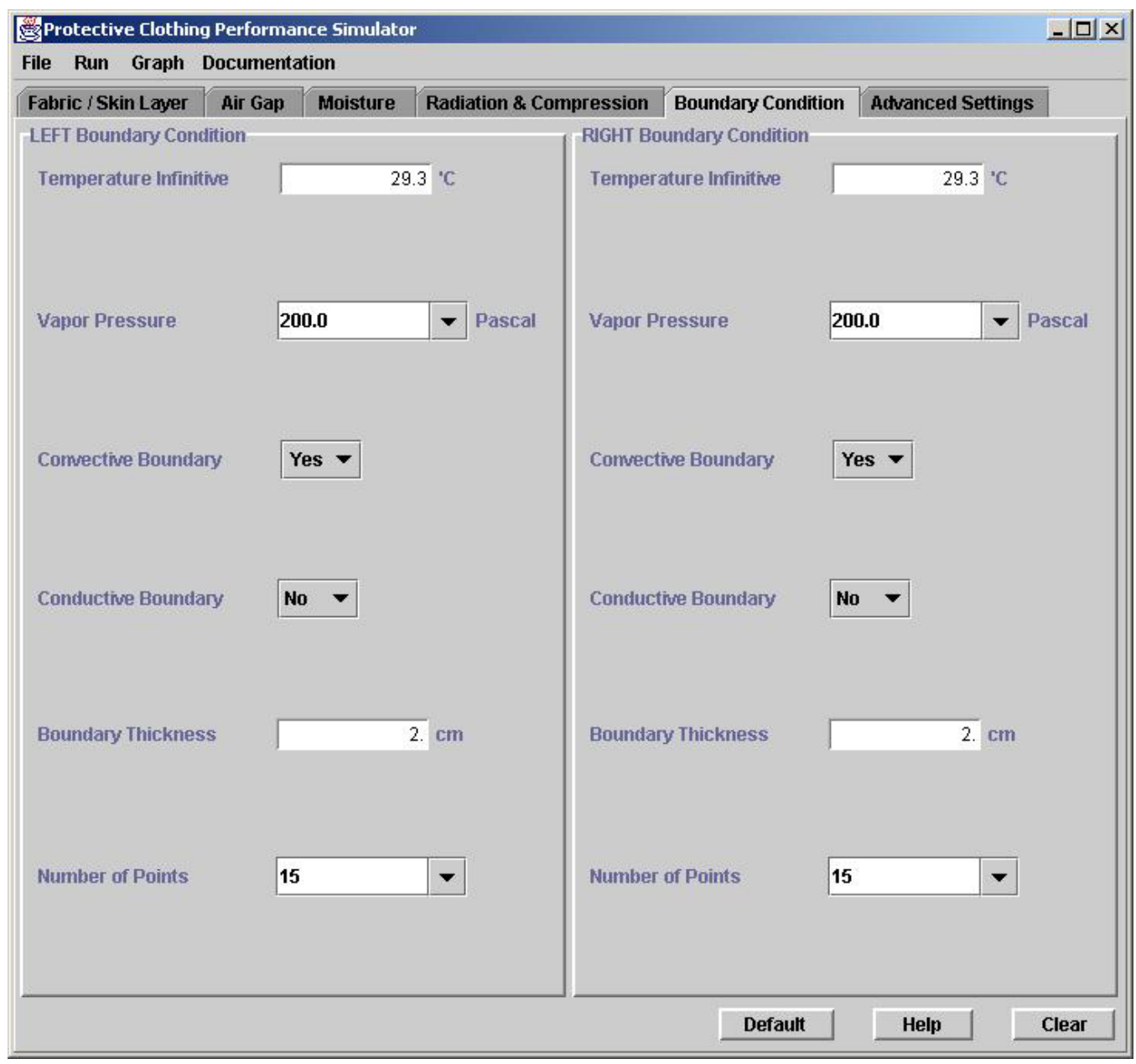

Figure 5.5: Boundary condition tab for dry fabric case study

The final tab that must be filled in for this scenario is the Advanced Settings tab. As the separate layers of skin are being modeled, the skin model pull down menu should be set to "True" and the body temperature of $37.0{ }^{\circ} \mathrm{C}$ entered. An initial time step of $0.00005 \mathrm{~s}$ can be entered either in the decimal format or in scientific format 5.0E-5.

A characteristic length of $30 \mathrm{~cm}$ is keyed into the appropriate box on the right hand portion of the screen. The fabric is vertically oriented by selecting "Vertical" from the Fabric Orientation pull-down. Material properties are considered to be temperature dependent from the Transport Property pull-down. No thermocouple output is required, and thus the Thermocouple Output is set to "No." The advanced settings tab should look as shown in Figure 5.6. 


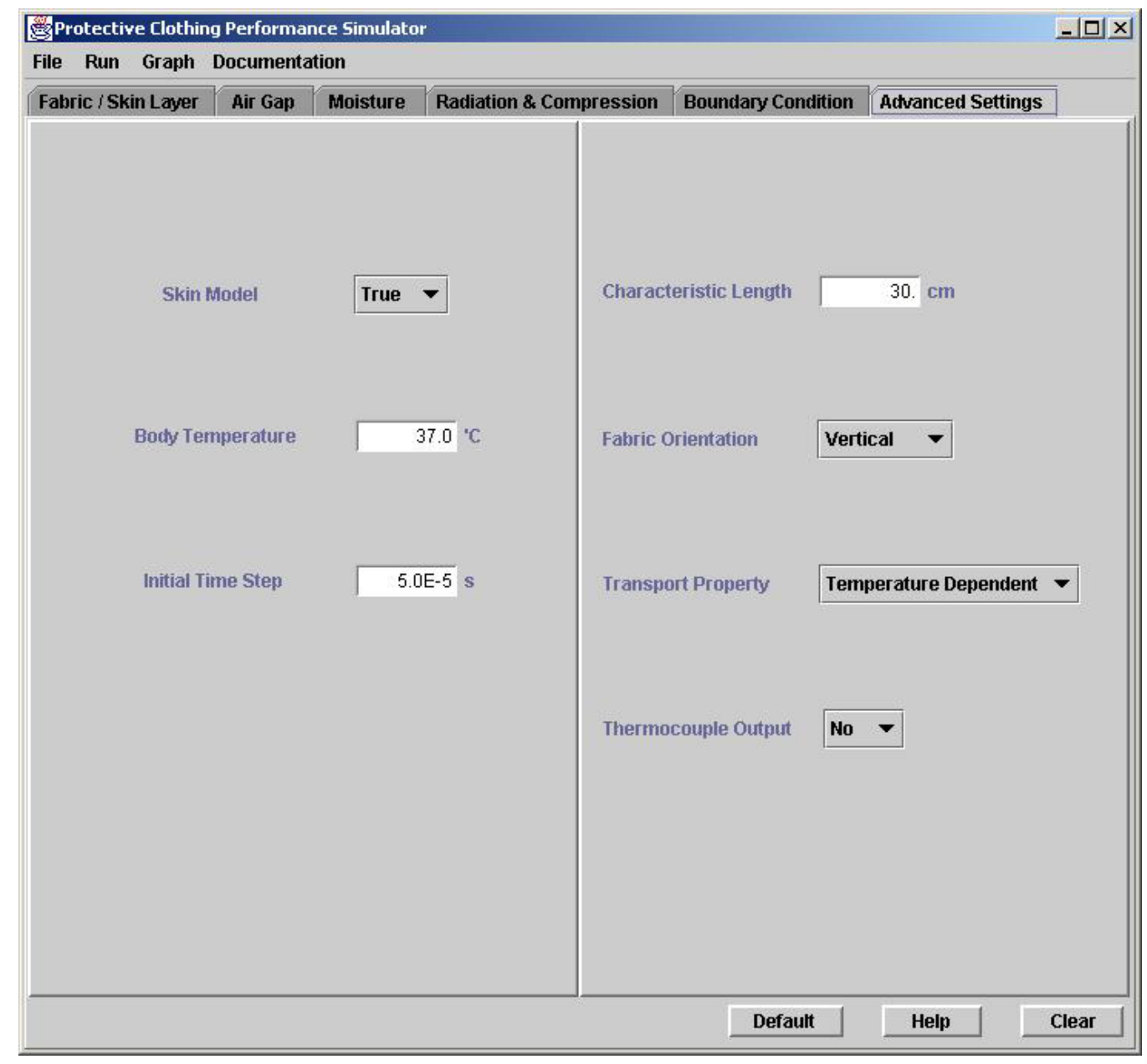

Figure 5.6: Advanced Settings tab for dry fabric case study

This fully defines the scenario under consideration. Each tab required a complete description of the problem as well as dummy values taken to satisfy the consistency check. Alternatively, this scenario corresponds to an example that is included with PCPS. The entire set of conditions can be imported by selecting the "Single layered fabric + skin, dry, low radiative flux" example (File, Import Examples, Single layered fabric + skin dry low radiative flux). A powerful means of defining a simulation is to import an example that has already been configured and then changing only those items desired by the user.

With the conditions so defined it is now possible to successfully execute the simulation (Run, execute). As the simulation runs, attention should be paid to the DOS shell status window. Between time 261.6 seconds and time 300 seconds, an alert message is printed to the window stating "First-degree Burn at $\mathrm{t}=278.6008$ s." This alert is shown in Figure 5.7. An alert such as this notifies the user that an important event occurred. 


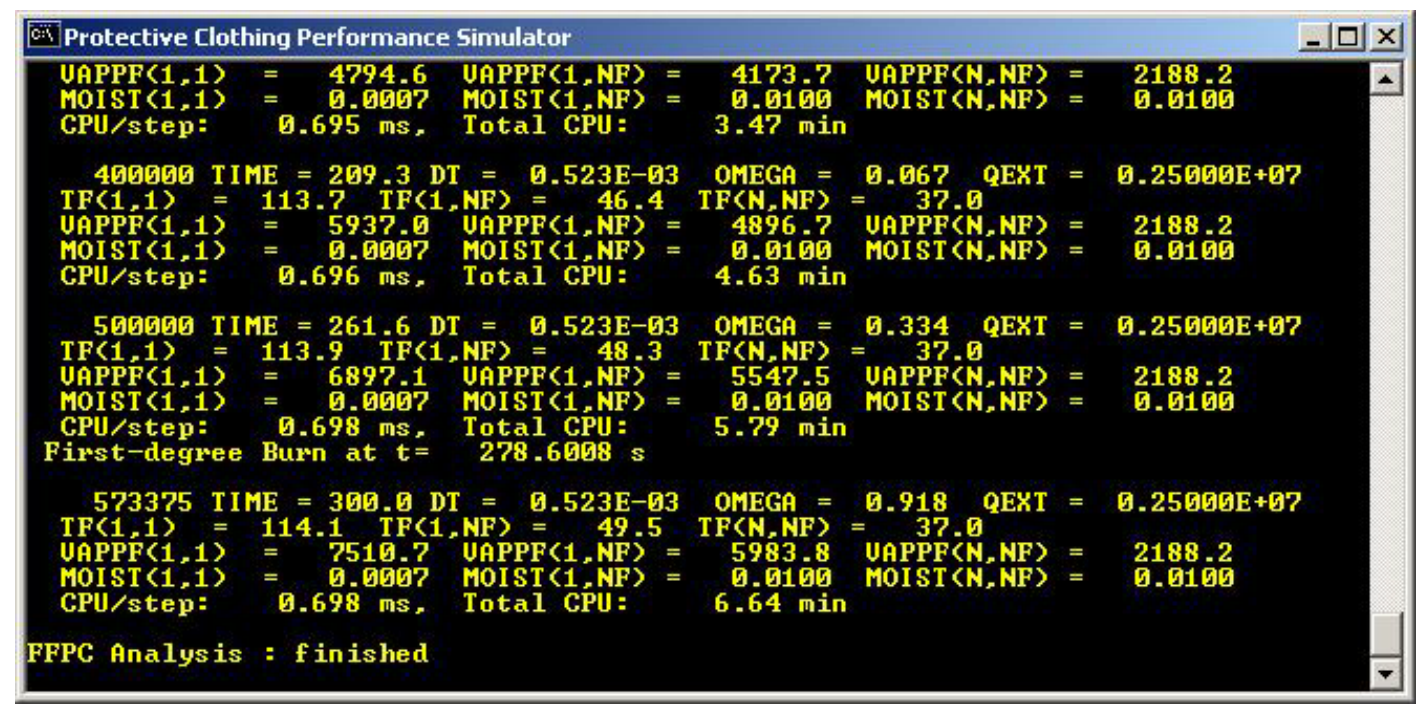

Figure 5.7: Dry fabric, status window

When the simulation concludes, the results are studied utilizing the graphs available through PCPS. The temperature of all of the layer faces (Graphs, Temperature (all faces) versus Time) is shown in Figure 5.8. The results show that the temperature at the left face rises quickly to a nearly steady temperature of $110{ }^{0} \mathrm{C}\left(230{ }^{0} \mathrm{~F}\right)$. The thermal barrier substantially slows the heat transfer through it so that the temperature at the right face of the fabric is significantly lower. Although still increasing slowly, at 300 seconds the right face of the fabric is approximately $70{ }^{\circ} \mathrm{C}\left(158{ }^{0} \mathrm{~F}\right)$.

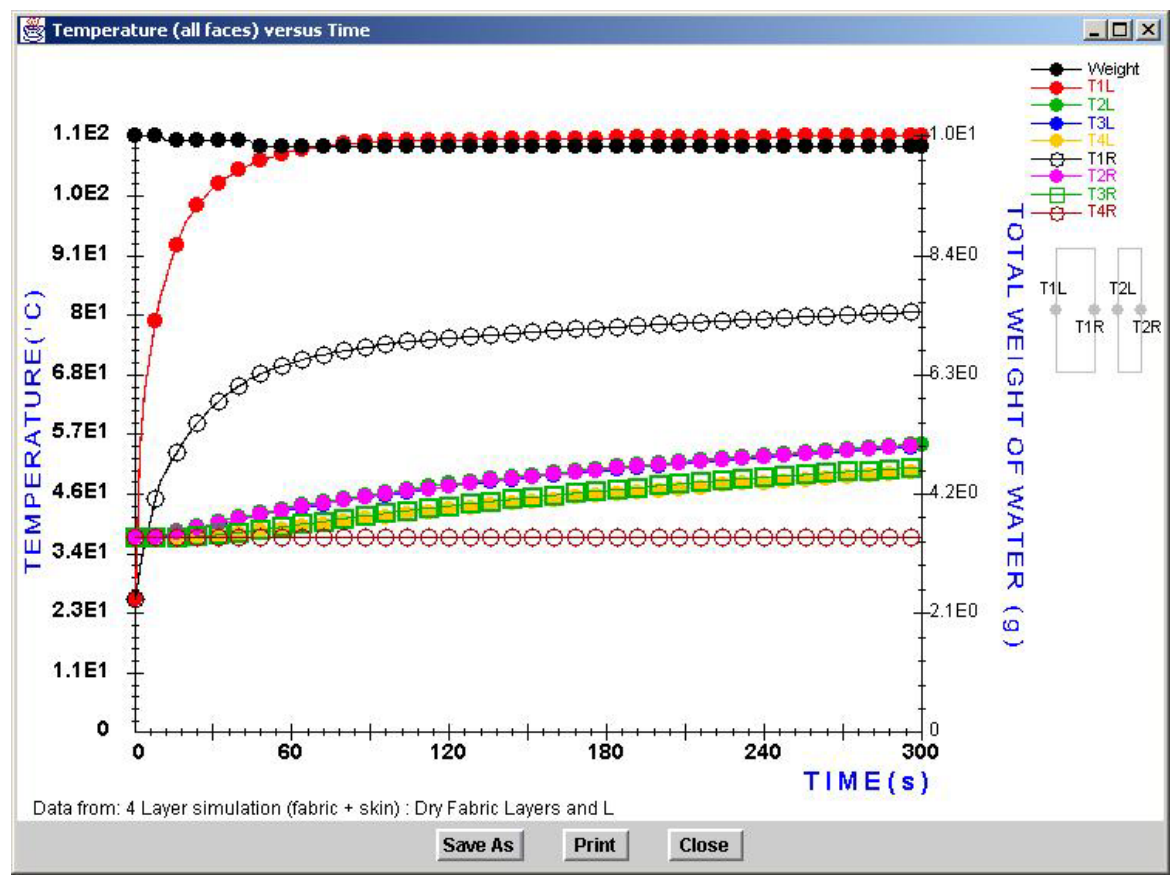

Figure 5.8: Dry fabric, temperature at all faces 
Heat is still being transferred through the fabric and into the skin however, and the graph shows that the temperature of the skin layers is subsequently increasing. Of the skin layers, the epidermal layer (T2L and $\mathrm{T} 2 \mathrm{R}$ ) temperature is nearly uniform and at 300 seconds is approximately $47^{\circ} \mathrm{C}\left(117^{\circ} \mathrm{F}\right)$. The temperature diminishes through each skin layer so that at the bottom of the subcutaneous tissue (T4R) the temperature is the core body temperature of $37{ }^{\circ} \mathrm{C}\left(98.6{ }^{\circ} \mathrm{F}\right)$.

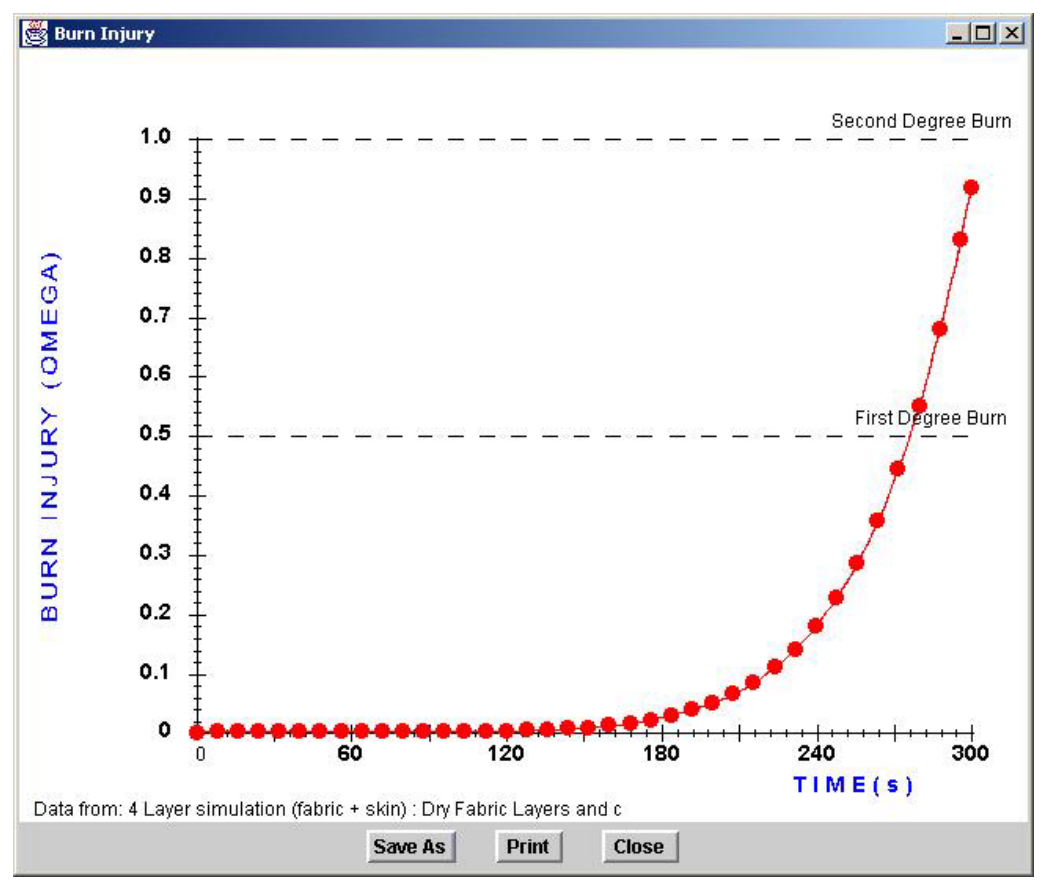

Figure 5.9: Dry fabric, burn injury

The possibility of tissue damage exists when the temperature of the skin layers is above the core body temperature. Indeed, the alert message from the status window stated that a first-degree burn was realized. The burn injury graph (Graphs, Burn Injury) shown in Figure 5.9 provides a measure of the amount of damage that occurred. At the beginning of the simulation, the injury function was near zero but begins to increase until at 278.6 seconds it crosses the "First Degree Burn" line (prompting the status window message). The curve continues to increase beyond this point so that at 300 seconds it had very nearly reached the "Second Degree Burn" limit. Had the simulation been allowed to proceed for any longer period of time, a second-degree burn would have resulted.

\subsection{Wet fabric}

The second scenario considered is similar however, now the fabric initially contains moisture. The initial moisture distribution is 2.0 grams (water) / grams (fabric) throughout the entire fabric. To define this case, a similar case is first imported. The 
user should select the case used to study the dry fabric in the previous example (File, Import Examples, Single layered fabric + skin dry low radiative flux). The only difference between this case and the case desired here is that the fabric layer is now taken to initially be wet.

To make the change the user should go to the moisture tab and change the values for fabric layer \#1 from the imported values of 0.01 to the new values of 2.0 grams (water) / grams (fabric). When completed, the moisture content tab should look as shown in Figure 5.10. Additionally, the user should change the title on the Fabric / Skin layer tab to adequately describe the conditions being simulated.

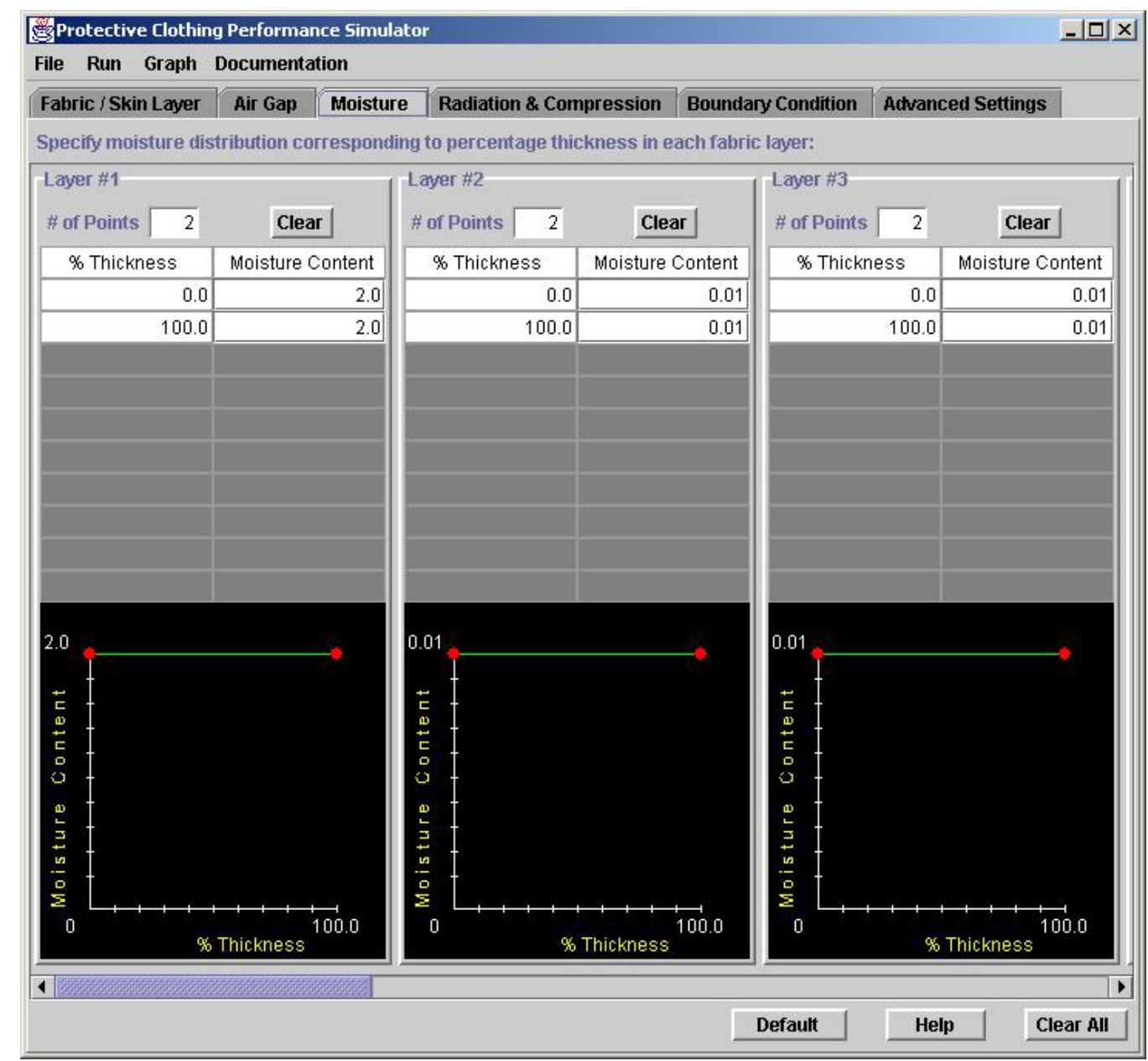

Figure 5.10: Moisture tab for wet fabric case study

With this change, the definition of conditions is complete and the simulation can be executed (Run, Execute). During the simulation, unlike the previous case, an alert message is not displayed in the status window. This means that a first-degree burn must not have occurred for this case. Part of the analysis should be to determine what produced the two different results. 
The graphs available through PCPS are again used to study the outcome of the simulation. Figure 5.11 shows the temperature of all the faces (Graphs, Temperature (all faces) versus Time) where a different behavior is seen as compared to the dry fabric. The left boundary (T1L) of the fabric does not rise as quickly when the fabric is wet. Additionally, the temperature difference between the left and right faces of the fabric is much smaller than that of the dry fabric. From the start of the simulation and for approximately the first 120 seconds, the left and right faces of the fabric rise at nearly the same rate. Beyond this point the left face begins to rise much faster and is still rising at 300 seconds. The temperature at this point is still significantly less than the previous scenario with a value of approximately $80 \mathrm{C}(176 \mathrm{~F})$. The right face of the fabric also shows a marked difference with a nearly steady temperature of approximately $54 \mathrm{C}$ (130 F).

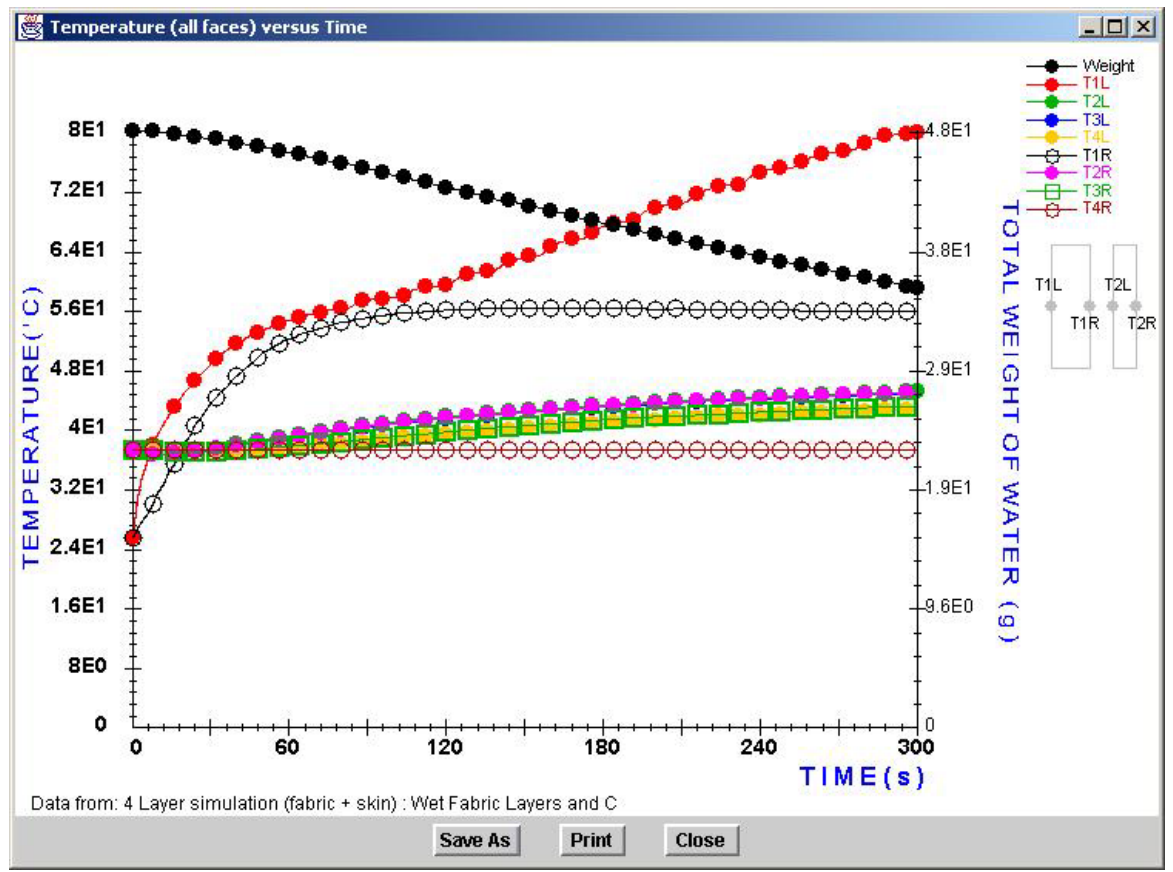

Figure 5.11: Wet fabric, temperature at all faces

With lower temperatures, the possibility of thermal injury is also lessened. In Figure 5.12 the burn injury graph for the case where the wet fabric is shown. Unlike before, the function never rises significantly above zero. No injury is found to occur for the simulation. 


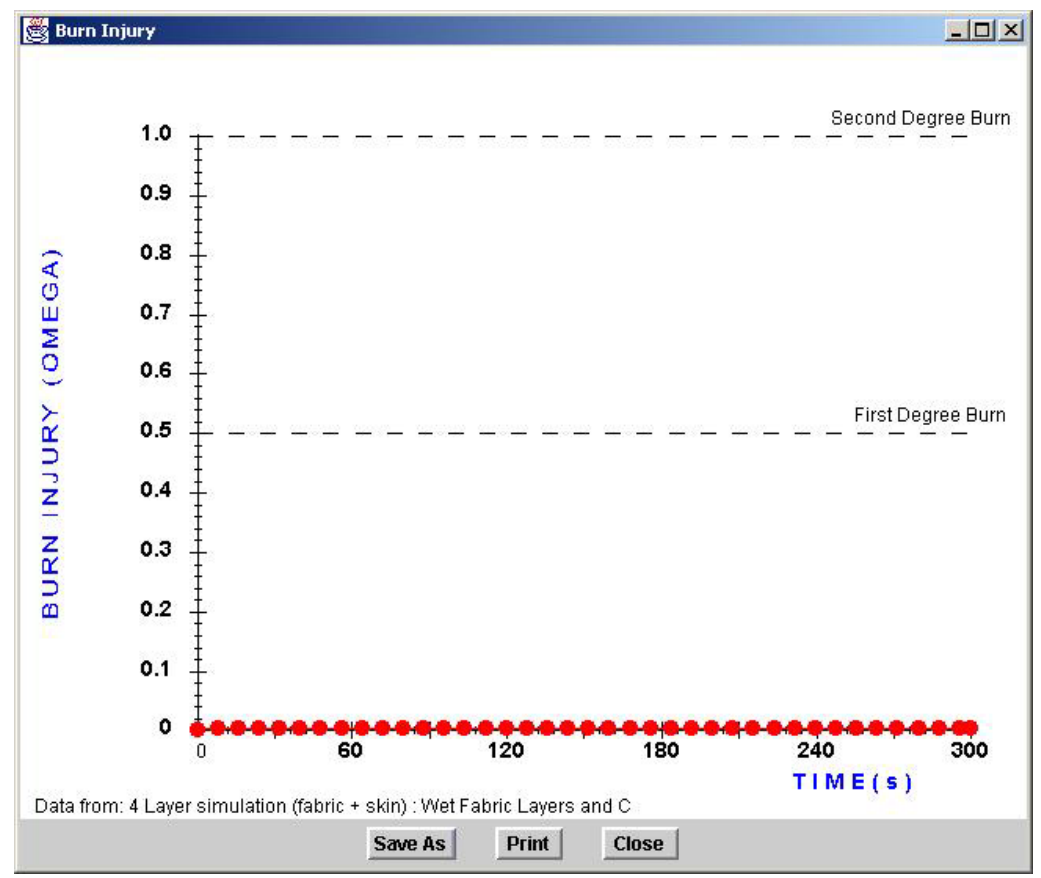

Figure 5.12: Wet fabric, burn injury

To determine the cause of the difference between the two cases, other graphs can be used. Additionally, this difference must somehow be related to the presence of moisture in the fabric. Figure 5.13 for the dry fabric and Figure 5.14 for the wet fabric show frames from the flow variable animations (Graphs, Animation of Flow Variables) at times of 100 seconds for each of the respective cases. For the dry graph a sharp slope exists between the front and back faces of the fabric, while for the wet fabric the temperature is nearly uniform. From the lower left graph of Figure 5.14 it is seen that at this point in time there still exists moisture across the entire fabric for the case of initially wet fabric. If the temperature and moisture distribution are considered for the wet fabric at a later time (Figure 5.15 shows the results at a time of 200 seconds) the moisture has evaporated from the left side of the fabric and there is a resulting increase in the temperature for this region. For the right side of the fabric, where there still exists a substantial amount of moisture, the temperature is nearly uniform. This describes the two significant effects that the inclusion of moisture had for the wet fabric considered here. The first effect distributed the temperature much more evenly across the fabric. The second result is that the evaporation of water acted to cool the fabric. Clearly, the presence of moisture within the fabric layers must be considered. 


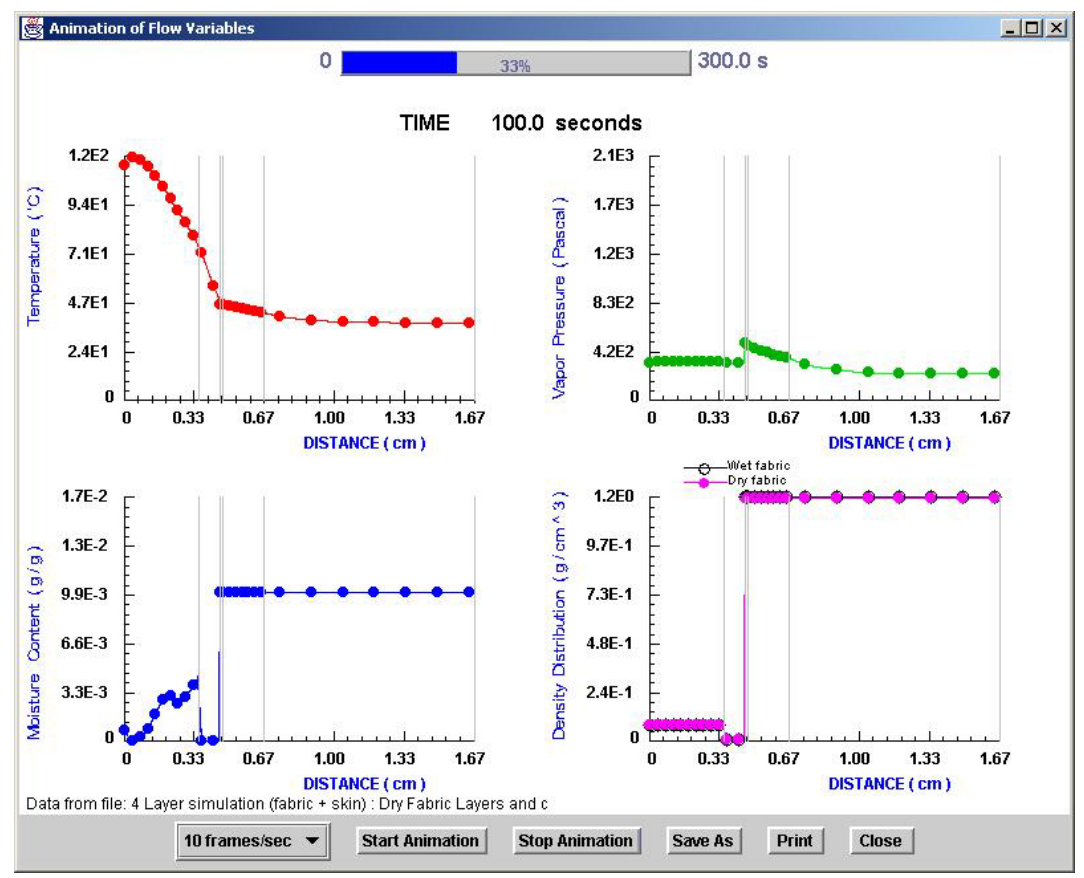

Figure 5.13: Dry fabric, flow variables at $t=100 \mathrm{~s}$

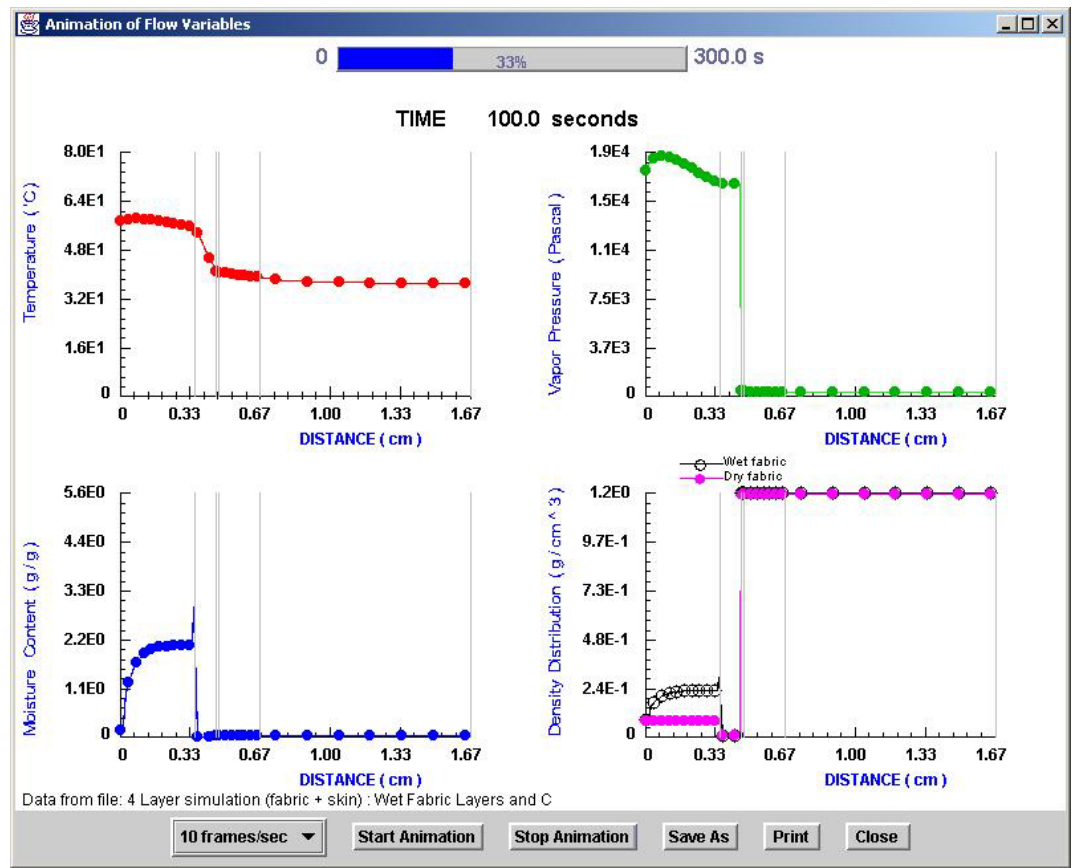

Figure 5.14: Wet fabric, flow variables at $t=100 \mathrm{~s}$ 


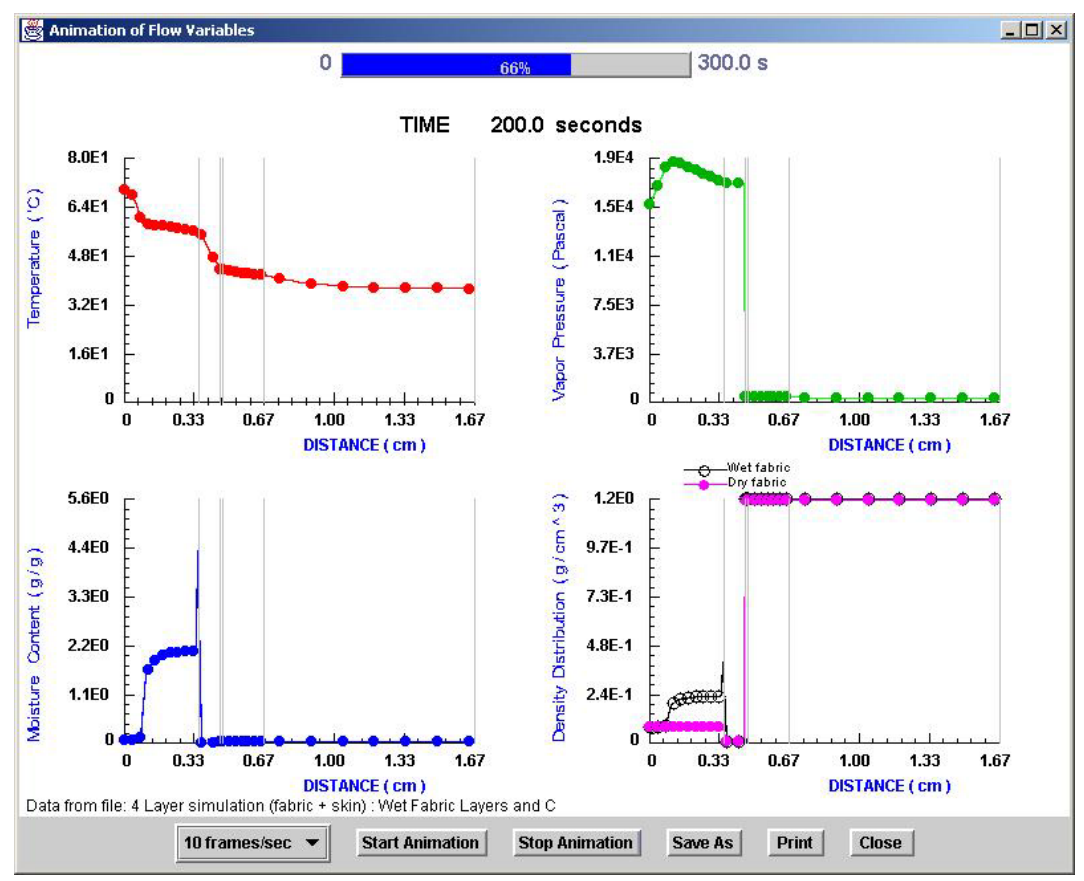

Figure 5.15: Wet fabric, flow variables at $t=200 \mathrm{~s}$ 


\section{Acknowledgements}

Appreciation is extended to Robert T. McCarthy of the United States Fire Administration for assistance provided during discussions related to fire fighter burn injuries and protective clothing performance. We thank J. Randall Lawson, Dan Madryzkowski, Robert Vettori, W. Twilley, W. Walton and Steve Kerber for their suggestions during the development phase and the beta test phase of the Graphical User Interface. Finally, the authors would like to thank Anthony Hamins, Dave Evans and Nelson Bryner for their overall support and guidance. 


\section{Appendix A : The predefined examples}

The following examples are available from within the GUI of PCPS. These correspond to INPUT files which are located in the "examples subdirectory. They can be loaded by selecting the appropriate case from Import Examples under the File pull-down menu.

1) Two layered fabric, dry, high radiative flux

2) Two layered fabric, dry, low radiative flux

3) Two layered fabric, dry, variable radiative flux

4) Two layered fabric, wet, high radiative flux

5) Two layered fabric, wet, low radiative flux

6) Two layered fabric, wet, variable radiative flux

7) Three layered fabric, dry, high radiative flux

8) Three layered fabric, dry, low radiative flux

9) Three layered fabric, dry, very high radiative flux

10) Three layered fabric, wet, high radiative flux

11) Three layered fabric, wet, low radiative flux

12) Three layered fabric, wet, very high radiative flux

13) Single layered fabric + skin, dry, high radiative flux

14) Single layered fabric + skin, dry, low radiative flux

15) Single layered fabric + skin, dry, variable radiative flux

16) Single layered fabric + skin, wet, high radiative flux

17) Single layered fabric + skin, wet, low radiative flux

18) Single layered fabric + skin, wet, variable radiative flux

19) Three layered fabric + skin, dry, high radiative flux

20) Three layered fabric + skin, dry, low radiative flux

21) Three layered fabric + skin, dry, variable radiative flux

22) Three layered fabric + skin, wet, high radiative flux

23) Three layered fabric + skin, wet, low radiative flux

24) Three layered fabric + skin, wet, variable radiative flux 


\section{A.1 Radiative flux definitions}

Low Radiative Flux

High Radiative Flux

Variable Radiative Flux

Very High Radiative Flux
$\mathrm{QR}=2.5 \mathrm{KW} / \mathrm{m} 2$

$\mathrm{QR}=25$ or $50 \mathrm{KW} / \mathrm{m} 2$ (case dependent)

Import file $=$ plume_tr.csv

$\mathrm{QR}=50 \mathrm{KW} / \mathrm{m} 2$

\section{A.2 Material, node and temperature definitions}

\section{TWO LAYERED FABRIC}

Layer 1: $\quad$ Kevlar Batting

$\mathrm{N}=20$

$\mathrm{T}=25.22 \mathrm{C}$

Layer 2:

Aramid

$\mathrm{N}=6$

$\mathrm{T}=25.22 \mathrm{C}$

THREE LAYERED FABRIC

Layer 1: $\quad$ Nomex III-Defender

$\mathrm{N}=20$

$\mathrm{T}=25.22 \mathrm{C}$

Layer 2: $\quad$ Neo-Guard

$\mathrm{N}=5$

$\mathrm{T}=25.22 \mathrm{C}$

Layer 3: $\quad$ Aralite

$\mathrm{N}=40$

$\mathrm{T}=25.22 \mathrm{C}$

SINGLE LAYERED FABRIC + SKIN

Layer 1:

Aralite

$\mathrm{N}=30$

$\mathrm{T}=25.22 \mathrm{C}$

Layer 2:

Epidermis

$\mathrm{N}=10$

$\mathrm{T}=37.0 \mathrm{C}$ 
Layer 3: Dermis

$\mathrm{N}=20$

$\mathrm{T}=37.0 \mathrm{C}$

Layer 4: $\quad$ Subcutaneous Tissue

$\mathrm{N}=20$

$\mathrm{T}=37.0 \mathrm{C}$

\section{THREE LAYERED FABRIC + SKIN}

Layer 1:

Nomex III-Defender

$\mathrm{N}=20$

$\mathrm{T}=23.7 \mathrm{C}$

Layer 2:

Neo-Guard

$\mathrm{N}=10$

$\mathrm{T}=23.7 \mathrm{C}$

Layer 3:

Aralite

$\mathrm{N}=10$

$\mathrm{T}=23.7 \mathrm{C}$

Layer 4:

Epidermis

$\mathrm{N}=10$

$\mathrm{T}=37.0 \mathrm{C}$

Layer 5:

Dermis

$\mathrm{N}=20$

$\mathrm{T}=37.0 \mathrm{C}$

Layer 6:

Subcutaneous Tissue

$\mathrm{N}=20$

$\mathrm{T}=37.0 \mathrm{C}$ 


\section{Appendix B : How to create your own INPUT file}

The GUI provides a structured means of entering the required information for a simulation. It conducts the necessary formatting of the file required by the Fortran solver and automatically performs a consistency check of the input. For these reasons, the GUI is the recommended method of entering simulation data.

The GUI is not a requirement however for using PCPS. Using just the Fortran code, a simulation can still be performed. On Windows ${ }^{\circledR}$, for example, a simulation can be performed by typing the command "FFPC.exe" from a command prompt set in the directory where PCPS is installed. While this performs the simulation, the interaction with the GUI for data input and the ability to visualize the graphs is obviously lost. This scenario may be desired if a faster, more high-powered machine is utilized to perform the calculations. Data can be copied back to a PC for post-processing.

This appendix lays out the rules for creating an INPUT file to perform an analysis. At the least, this should allow a user to be able to edit an already existing INPUT file to make minor changes without the need for going into the GUI. It is assumed that the reader is familiar with the GUI input.

The basic rules for creating an INPUT file are as follows:

1) There are no blank lines at the beginning or between subsequent lines of data

2) Each line corresponds to specific data

3) Comments may be placed at the end of a line (after the data) but no comment extends to the next line.

The general layout of the INPUT file follows closely the layout of the input tabs,

----START OF INPUT FILE-----
CONTROL BLOCK
FABRIC / SKIN LAYER BLOCK
MOISTURE BLOCK
SKIN BLOCK
AIR GAP BLOCK
BOUNDARY CONDITION BLOCK
ADVANCED SETTINGS BLOCK
----END OF INPUT FILE-----


The format of each block is provided in the following sections. To aid in understanding the formatting, line numbers have been inserted for clarity but are not to be inserted at the beginning of the true INPUT file. When an INPUT file is created through the GUI, comments are automatically placed after each of the data lines to aid in decrypting the information contained.

For those users wishing to use this form of data entry, the example INPUT files stored in the "examples" subdirectory would be a good place to start. These files can be imported to the GUI, and the file studied alongside the input tabs to see how specific features relate. Also remember that when using the GUI, an INPUT file is created when the Execute command is selected. This will overwrite any previously existing files. It is recommended therefore to name the file to be imported something other than INPUT.

\section{B.1 CONTROL BLOCK}

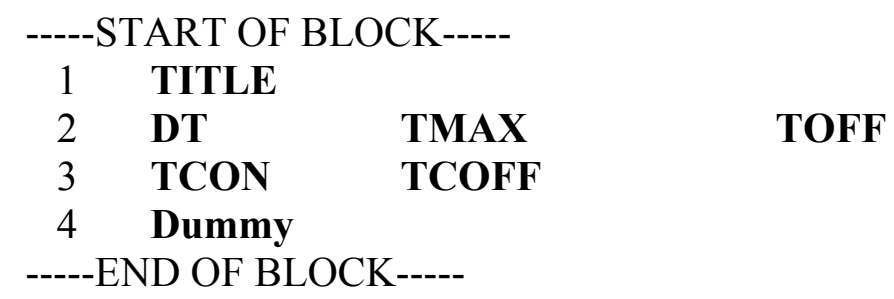

In the above block, the following definitions apply,

TITLE A name for the simulation, placed in every output data file

DT Initial time step (seconds)

TMAX Maximum time for simulation (seconds)

TOFF Cut-off time for external radiation (seconds)

TCON Beginning time for external compression

TCOFF Ending time for external compression

Dummy Dummy string for compression file

\section{B.2 FABRIC / SKIN LAYER BLOCK}

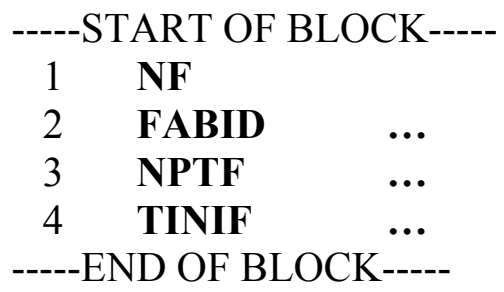

In the above block, the following definitions apply, 
NF Number of fabric layers

FABID Fabric ID

NPTF Nodes in fabric layer

TINIF Initial temperature of fabric layer (degrees Celsius)

... Continuation ellipses for multiple layers. Data is separated by a space

The fabrics are entered from left to right in sequential order. The possible fabrics are the same as those available from the pull-down menus of the GUI. Table B.1 lists the fabric ID's corresponding to the specific fabrics available.

\begin{tabular}{|c|c|}
\hline $\begin{array}{c}\text { Fabric ID } \\
(\text { FID) }\end{array}$ & Fabric Material \\
\hline 1 & Aralite $^{(B)}$ \\
\hline 2 & Breathe-Tex ${ }^{\circledR}$ \\
\hline 3 & Breathe-Tex ${ }^{\circledR}$ Plus \\
\hline 4 & Cotton Duck \\
\hline 5 & Nomex ${ }^{\circledR}$ E-89 Crosstech ${ }^{\circledR}$ \\
\hline 6 & Neo-Guard ${ }^{\circledR}$ \\
\hline 7 & Nomex ${ }^{\circledR}$ III-Defender ${ }^{\mathrm{TM}}$ \\
\hline 8 & Nomex ${ }^{\circledR}$ IIIA Pajama Check Crosstech ${ }^{\circledR}$ \\
\hline 9 & PBI $^{\mathrm{TM}}$ Kevlar $^{\circledR}$ Kombat $^{\mathrm{TM}}$ \\
\hline 10 & Scotchlite ${ }^{\circledR}$ \\
\hline 11 & Epidermis \\
\hline 12 & Dermis \\
\hline 13 & Subcutaneous Tissue \\
\hline 14 & Kevlar $^{\circledR}$ Batting \\
\hline 15 & Aramid \\
\hline 16 & Aralite $^{\circledR}-1$ \\
\hline 17 & Kevlar $^{\circledR}$ Batting -1 \\
\hline 18 & Aramid -1 \\
\hline
\end{tabular}

Table B.1: Fabric ID numbers for available fabrics

\section{B.3 MOISTURE BLOCK}

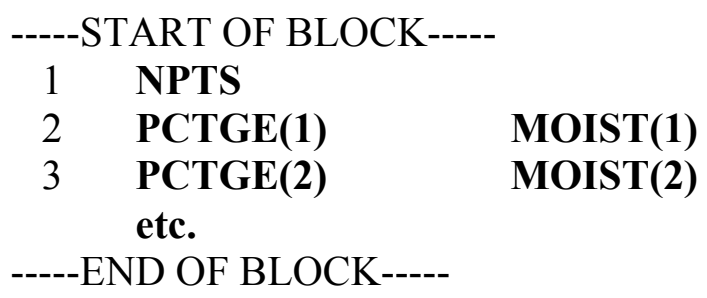

In the above block, the following definitions apply, 
NPTS Number of points used to define the moisture distribution for a layer PCTGE(i) Location (percentage) of moisture distribution at $i^{\text {th }}$ point of a layer

MOIST(i) Moisture distribution at $\mathrm{i}^{\text {th }}$ point of a layer

etc. Additional layers continue with new lines

It is necessary to include information for each layer of fabric defined. The first moisture set is for layer one, the second set for layer two, etc.

\section{B.4 SKIN BLOCK}

\section{-----START OF BLOCK----- \\ 1 INCLUDE BODYT \\ -----END OF BLOCK-----}

In the above block, the following definitions apply,

INCLUDE A Boolean value stating whether or not to include a skin model

BODYT Body temperature (degrees Celsius)

The Boolean value for the skin model follows the Fortran convention and takes values of

'.True.' or '.False.' (note the periods). If the fabric layers contains skin layers, then the skin model must be '.True.' otherwise this value is '.False.'

\section{B.5 AIR GAP BLOCK}

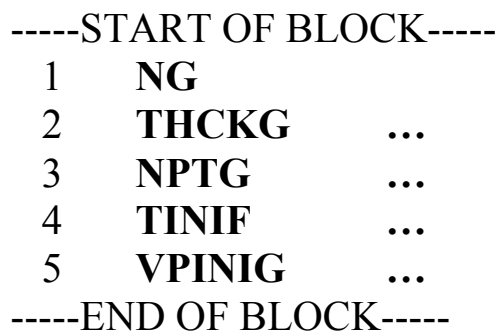

In the above block, the following definitions apply,

NG Number of air gaps

THCKG Thickness of air gap (centimeters)

NPTG Nodes in air gap

TINIF Initial temperature of air gap (degrees Celsius)

VPINIG Vapor pressure of air gap (Pascal)

Continuation for multiple points. Data is separated by a space 
The number of air gaps is dictated by the fabric layers entered in the FABRIC / SKIN LAYER BLOCK, and must therefore be equal to the number of fabric / skin layers minus one. This entry is calculated automatically in the GUI. Data begins with the left gap (between material layers one and two) and progresses sequentially to the right.

\section{B.6 BOUNDARY CONDITION BLOCK}

\begin{tabular}{|c|c|c|}
\hline & $\Gamma 0$ & K---. \\
\hline 1 & TINFL & TINFR \\
\hline 2 & VPINFL & VPINFR \\
\hline 3 & RADS & \\
\hline 4 & QEXT & \\
\hline 5 & IBCL & IBCR \\
\hline 6 & IAIRL & IAIRR \\
\hline 7 & THCKBL & THCKBR \\
\hline 8 & NPTBL & NPTBR \\
\hline
\end{tabular}

In the above block, the following definitions apply,

TINFL Left ambient temperature (degrees Celsius)

TINFR Right ambient temperature (degrees Celsius)

VPINFL Left boundary vapor pressure (Pascal)

VPINFR Right boundary vapor pressure (Pascal)

RADS Switch for radiative flux condition $(0=$ constant, $1=$ variable $)$

QEXT External incident radiative heat flux (erg / seconds-centimeter $\left.{ }^{2}\right)^{*}$

IBCL Use convective boundary conditions on left $(0=$ no, $1=$ yes $)$

IBCR Use convective boundary conditions on $\operatorname{right}(0=$ no, $1=$ yes $)$

IAIRL Use conductive boundary conditions on left $(0=$ no, $1=$ yes $)$

IAIRR Use conductive boundary conditions on right $(0=$ no, $1=$ yes $)$

THCKBL Left gas boundary thickness (centimeters)

THCKBR Right gas boundary thickness (centimeters)

NPTBL Number of nodes used for the left gas boundary

NPTBR Number of nodes used for the right gas boundary

* For constant heat radiative fluxes, this entry should be the flux value. For variable radiative fluxes this entry is the file name to be used for specifying the variable fluxes.

\section{B.7 ADVANCED SETTINGS BLOCK}

$\begin{array}{ll}\text {----START OF BLOCK----- } \\ 1 & \text { XL } \\ 2 & \text { IPLATE }\end{array}$




\section{ITHRMC \\ 4 IVAIR \\ -----END OF BLOCK-----}

In the above block, the following definitions apply,

XL Characteristic length scale (centimeters)

IPLATE Orientation of fabric $(0=$ horizontal, $1=$ vertical $)$

ITHRMC Thermal transport property $(0=$ constant, $1=$ temperature dependent $)$

IVAIR Thermocouple output $(0=$ no, $1=$ yes $)$

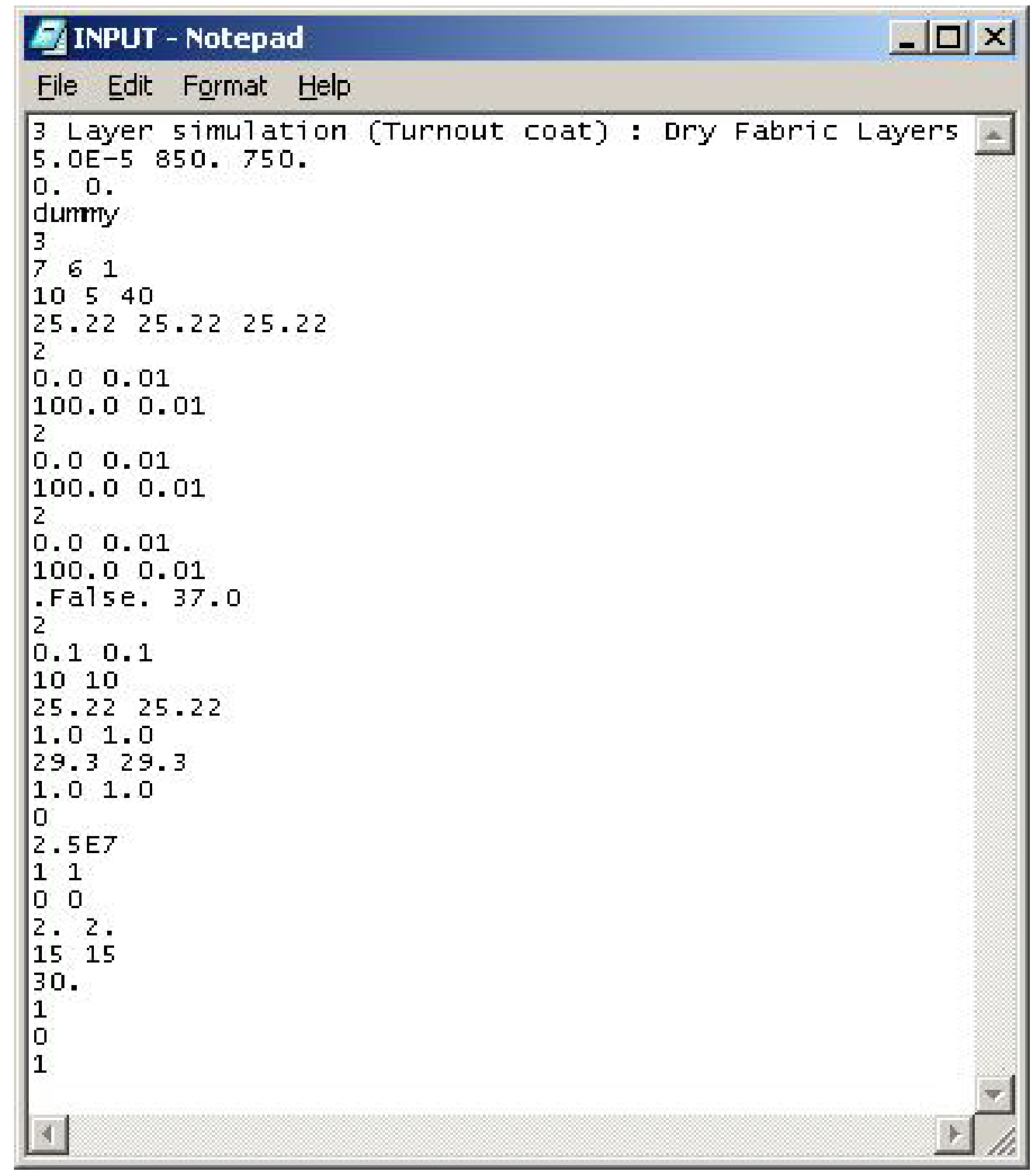

Figure B.5.16: A sample INPUT file 